\title{
Stimulus-locked responses of two phase oscillators coupled with delayed feedback
}

\author{
Valerii Krachkovskyi, ${ }^{1}$ Oleksandr V. Popovych, ${ }^{1}$ and Peter A. Tass ${ }^{1,2}$ \\ ${ }^{1}$ Institute of Medicine and Virtual Institute of Neuromodulation, Research Centre Jülich, 52425 Jülich, Germany \\ ${ }^{2}$ Department of Stereotaxic and Functional Neurosurgery, University Hospital, 50924 Cologne, Germany and Brain Imaging, \\ Center West, 52425 Jülich, Germany
}

(Received 9 March 2006; published 23 June 2006)

\begin{abstract}
For a system of two phase oscillators coupled with delayed self-feedback we study the impact of pulsatile stimulation administered to both oscillators. This system models the dynamics of two coupled phase-locked loops (PLLs) with a finite internal delay within each loop. The delayed self-feedback leads to a rich variety of dynamical regimes, ranging from phase-locked and periodically modulated synchronized states to chaotic phase synchronization and desynchronization. Remarkably, for large coupling strength the two PLLs are completely desynchronized. We study stimulus-locked responses emerging in the different dynamical regimes. Simple phase resets may be followed by a response clustering, which is intimately connected with long poststimulus resynchronization. Intriguingly, a maximal perturbation (i.e., maximal response clustering and maximal resynchronization time) occurs, if the system gets trapped at a stable manifold of an unstable saddle fixed point due to appropriately calibrated stimulus. Also, single stimuli with suitable parameters can shift the system from a stable synchronized state to a stable desynchronized state or vice versa. Our result show that appropriately calibrated single pulse stimuli may cause pronounced transient and/or long-lasting changes of the oscillators' dynamics. Pulse stimulation may, hence, constitute an effective approach for the control of coupled oscillators, which might be relevant to both physical and medical applications.
\end{abstract}

DOI: 10.1103/PhysRevE.73.066220

PACS number(s): 05.45.Xt, 87.19.La

\section{INTRODUCTION}

Transient responses of coupled oscillators to pulsatile stimuli are relevant in several fields of the natural sciences [1-4]. Such responses are typically studied by experimentalists to obtain information on dynamical systems and to characterize the system's inventory of reactions. For example, transient short-term brain responses evoked by sensory stimuli play a key role in the study of cerebral information processing $[5,6]$. Furthermore, stimulus evoked responses as measured with electroencephalography (EEG) are a standard tool for neurological diagnosis [7]. A stimulus-locked response of a neuronal population is typically analyzed with a cross-trial averaging (CTA), where an ensemble of poststimulus responses is averaged across trials $[5,8]$.

Several studies indicate that brain oscillations play a major role in the generation of stimulus-locked EEG responses. For instance, such responses are considered to be caused by a phase reset of the alpha rhythm (in the $10 \mathrm{~Hz}$ frequency range) [9-11]. The occurrence of a phase reset of a brain rhythm means that the phase of this oscillation attains a preferred value at a particular time relative to stimulus offset [1-4], which can be detected and quantified by means of phase resetting indices $[4,11-18]$.

However, recently it has been shown that phase oscillators with instantaneous coupling may not just undergo a stimuluslocked phase reset, but may react to pulsatile stimuli in a more complex manner [14-18]. Apart from simple phase resets such stimuli may elicit a response clustering, that means, a switching between qualitatively different, in particular, antiphase responses across trials: At a particular time relative to stimulus offset the phase of an oscillator attains two or more preferred values across trials. This phenomenon may by itself be interesting. However, even more important is that response clustering can typically neither be detected with CTA nor with typical phase resetting indices as defined by Refs. [11-13] (compare Refs. [14-18]).
In other words, the standard tools for the analysis of transient neural responses are not sensitive to this particular well-defined type of stimulus-locked responses. For this reason, a stochastic phase response analysis has been developed which allows to detect phase resets as well as response clustering $[4,14-18]$.

Next, the question arises whether response clustering and possibly even more complex types of stimulus-locked responses occur in more complex oscillator models. If so, we would like to know whether the data analysis available enables us to detect such responses. Previous studies were performed in coupled phase oscillators without any time delay [14-18]. Delays are inevitable in natural systems and may occur within and between oscillators [19-27]. It has been shown that delays may severely alter the spontaneously emerging dynamics of coupled oscillators [28-37]. Accordingly, it is important to take into account the impact of delays also on stimulus-locked responses.

We here study stimulus-locked responses of two coupled phase-locked loops. A phase-locked loop (PLL) is a generic device which may act with an internal delay and which is used for adjusting its phase to the phase of an external reference signal [38-40]. PLLs are widely used in electrical engineering [38] and can be implemented by software as well as hardware [41]. Furthermore, PLLs can be found in different parts and on different levels of the nervous system: Both single neurons [42] and neuronal circuits [43,44] may function as a PLL. In fact, several important neuronal circuits can be modeled by means of PLLs, for instance, in the context of motor control [40,45] or sensory decoding [44,46-48]. We study two nearly identical PLLs coupled without delay. In other words, we couple these generic structures, assuming that, in a first approximation, we can neglect the (transmission) delay between the PLLs compared to the PLLs' internal delay. 


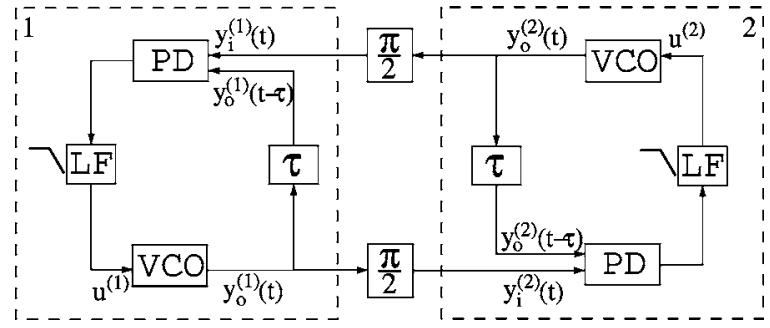

FIG. 1. Schematic picture of an electrical circuit of two interacting phase-locked loops with internal delay. The subscripts " $i$ " and " $o$ " stand for input and output signals, respectively.

Our analysis reveals several qualitatively different dynamical regimes of the coupled PLLs, which emerge spontaneously, i.e., in the absence of stimulation, depending on model parameters. Apart from unistable dynamical regimes we also observe multistability for a wide range of parameters. Furthermore, depending on the dynamical regime of the stimulated system, its response to a stimulus can follow completely different scenarios. In the phase-locked regime a response clustering with two clusters occurs, comparable to what has been found for two phase oscillators coupled without delay $[14-16,18]$. In contrast, in the regime of periodically modulated phase synchronization we observe a response clustering with stimulus-locked, time-varying configuration: Relative to stimulus onset the ensemble of responses oscillates between states of a pronounced phase reset and states with response clustering (with two antiphase response clusters). Moreover, in the multistable regime stimulation can induce a switching between different stable synchronized states. In this way, a single-shot stimulus can even completely change the type of the long-term dynamics of the system, e.g., by shifting the system from a synchronized to a desynchronized state.

We show that the time delay does not only cause a variety of spontaneous dynamical regimes and corresponding transient responses. Rather, fundamental dynamical features, such as the recovery after stimulation, may be completely different compared to the case without time delay. A delayed self-feedback leads to a pronounced vulnerability to appropriate stimuli. The latter prepare the system in a way that it gets trapped in the infinite dimensional phase space, so that the time it takes to recover (especially, to resynchronize) increases in a nearly singular manner.

The paper is organized as follows. We introduce our model equation in Sec. II. In Sec. III we present a short description of the model's dynamics, which emerges spontaneously. Stimulus-locked dynamics during stimulation, the so-called "in-stimulus dynamics," is studied in Sec. IV, whereas Secs. V and VI are devoted to the "post-stimulus dynamics," the transient dynamics evolving after stimulus offset, in unistable as well as multistable dynamical regimes. We summarize and discuss our results in Sec. VII.

\section{MODEL EQUATION}

The dynamical equations of two coupled PLLs can be derived based on the scheme shown in Fig. 1. A phase- locked loop is a device by means of which the phase of a frequency-modulated oscillator output is obliged to follow that of the input signal. It consists in general of three blocks: phase detector (PD), loop filter (LF), and voltage-controlled oscillator (VCO) [38]. Two signals are applied to a PD, the output of which is a function of the phase difference between the two signals applied. This error voltage, after being lowpass filtered by the LF, is fed into the VCO in such a way that the oscillator signal phase must follow the input signal phase.

Let us consider the first PLL in Fig. 1 (left block). The output signal $y_{0}^{(1)}(t)=A \cos \left[\psi_{1}(t)\right]$ of the VCO with constant amplitude $A$ and time dependent phase $\psi_{1}(t)$, is retarded with a delay $\tau$. The superscript "(1)" refers to PLL1. The delayed signal $y_{0}^{(1)}(t-\tau)=A \cos \left[\psi_{1}(t-\tau)\right]$ is then applied to the phase detector together with an external input signal of the form $y_{i}^{(1)}(t)=B \sin \left[\psi_{2}(t)\right]$. The PD of the PLL has a sinusoidal characteristic, producing an output signal which is proportional to the product of the two incoming signals $y_{i}^{(1)}(t) y_{0}^{(1)}$ $\times(t-\tau)$. The next element of the PLL circuit is a low-pass filter which eliminates high-frequency components, such that the resulting signal $u^{(1)}$ attains the form $u^{(1)}(t)$ $=K / 2 \sin \left[\psi_{2}(t)-\psi_{1}(t-\tau)\right]$, see also Refs. [38,39]. Finally, the signal $u^{(1)}(t)$ is applied to the VCO which adjusts its instantaneous angular frequency proportionally to the incoming signal around its central angular frequency $\omega_{1}: \dot{\psi}_{1}(t)$ $=\omega_{1}+u^{(1)}(t)=\omega_{1}+K / 2 \sin \left[\psi_{2}(t)-\psi_{1}(t-\tau)\right]$.

The VCO output signal $y_{0}^{(1)}(t)$ is not only used within PLL1, but is also sent to PLL2 (see Fig. 1). Passing through the interconnecting block denoted by $\frac{\pi}{2}$ in Fig. 1, the signal's phase is shifted by a quarter of a period. This phase shifted signal then serves as input signal for PLL2: $y_{i}^{(2)}(t)$ $=A \sin \left[\psi_{1}(t)\right]$. The same derivation as above can be applied to PLL 2, and we obtain the following equation for the phase $\psi_{2}$ of the second PLL: $\dot{\psi}_{2}(t)=\omega_{2}+K / 2 \sin \left[\psi_{1}(t)-\psi_{2}(t-\tau)\right]$, where $\omega_{2}$ is the central frequency of the second VCO. Therefore, the phase dynamics of the two coupled PLLs with the internal delay $\tau$ can be modeled by the following system of two phase oscillators with time-delayed coupling:

$$
\begin{aligned}
& \dot{\psi}_{1}(t)=\omega_{1}+\frac{K}{2} \sin \left[\psi_{2}(t)-\psi_{1}(t-\tau)\right], \\
& \dot{\psi}_{2}(t)=\omega_{2}+\frac{K}{2} \sin \left[\psi_{1}(t)-\psi_{2}(t-\tau)\right],
\end{aligned}
$$

where parameter $K$ plays a role of a coupling parameter (or loop gain of PLLs [38]). $\omega_{i}, i=1,2$ are the natural frequencies of the oscillators, and $\tau$ is a time delay. According to Eq. (1) the oscillators are coupled in a such a way that the timedelayed self-feedback from each oscillator is combined with an instantaneous signal from the other oscillator, respectively. So, the output signal of each PLL serves as input signal for the other PLL. In this way, each PLL serves as external reference to which the other PLL has to adapt. Note, we assume that the interaction between the PLLs is fast compared to the internal delay $\tau$. Accordingly, in a first approxi- 
mation we neglect external delays which may occur when the output signal of a VCO of one PLL is sent to the PD of the other PLL. The opposite case would be to neglect the internal delay and take into account only a transmission delay between the PLLs. In that case we obtain a system of two coupled phase oscillators with transmission delay, which has been studied by Schuster and Wangner [28].

We study responses of system (1) to strong external stimuli $S_{j}$ administered to each oscillator $(j=1,2)$. Furthermore, for both oscillators we introduce random forces $F_{j}$, modeled by additive Gaussian white noise. In this way, the following combined driving terms $Y_{j}(t), j=1,2$, are added to the right-hand side of Eq. (1), respectively,

$$
Y_{j}(t):=X(t) I \cos \left(\psi_{j}-\theta_{j}\right)+F_{j}(t)
$$

(compare Refs. [14-16,18]). $S_{j}(t)=X(t) I \cos \left(\psi_{j}-\theta_{j}\right)$ models the external stimulation of the $j$ th oscillator. $X(t)=1$ or 0 if the stimulus is on or off, respectively. $I$ is the stimulus intensity, $\theta_{j}$ are stimulation phase shifts. $F_{i}(t)$ is a Gaussian $\delta$-correlated noise with $\left\langle F_{j}(t)\right\rangle=0$ and $\left\langle F_{i}(t) F_{j}(\widetilde{t})\right\rangle=D \delta_{i j} \delta(t$ $-\widetilde{t}$ ), where $D$ is a constant noise amplitude. Without loss of generality, in this paper we consider the case $\theta_{1}=0$, i.e., only the second oscillator $\psi_{2}$ is stimulated with the phase shift $\theta$ $=\theta_{2} \in[0,2 \pi]$ which turns out to be a main stimulation parameter. The stimulation protocol is explained in Sec. IV.

\section{SPONTANEOUS DYNAMICS OF THE MODEL}

In this section we briefly present the main dynamical regimes of the two coupled phase oscillators with delay (1) which emerge spontaneously in the absence of stimulation. A more detailed study of dynamics of system (1) is reported elsewhere $[36,49]$.

We introduce the phase difference $\varphi_{1}$ and the mean phase $\varphi_{2}$

$$
\begin{gathered}
\varphi_{1}(t)=\psi_{2}(t)-\psi_{1}(t), \\
\varphi_{2}(t)=\left[\psi_{2}(t)+\psi_{1}(t)\right] / 2,
\end{gathered}
$$

so that system (1) reads

$$
\begin{aligned}
\dot{\varphi}_{1}(t)= & \Delta_{1}-K \sin \left(\frac{\varphi_{1}(t)+\varphi_{1}(t-\tau)}{2}\right) \\
& \times \cos \left[\varphi_{2}(t)-\varphi_{2}(t-\tau)\right], \\
\dot{\varphi}_{2}(t)= & \Delta_{2}+\frac{K}{2} \cos \left(\frac{\varphi_{1}(t)+\varphi_{1}(t-\tau)}{2}\right) \\
& \times \sin \left[\varphi_{2}(t)-\varphi_{2}(t-\tau)\right] .
\end{aligned}
$$

Here, $\Delta_{1}=\omega_{2}-\omega_{1}$ is the natural frequency mismatch, and $\Delta_{2}=\left(\omega_{2}+\omega_{1}\right) / 2$ is the mean natural frequency. In the uncoupled regime for $K=0$ each phase $\psi_{j}$ in system (1) grows with its own frequency $\omega_{j}$ such that the phase difference $\varphi_{1}(t)$ has the frequency $\Delta_{1}$. A desynchronized dynamics, where the phase difference exhibits unbounded rotations, is preserved in system (4) for small values of $\mathrm{K}$ provided that $\Delta_{1} \neq 0$. This is shown in Fig. 2(a), where an exemplary one-
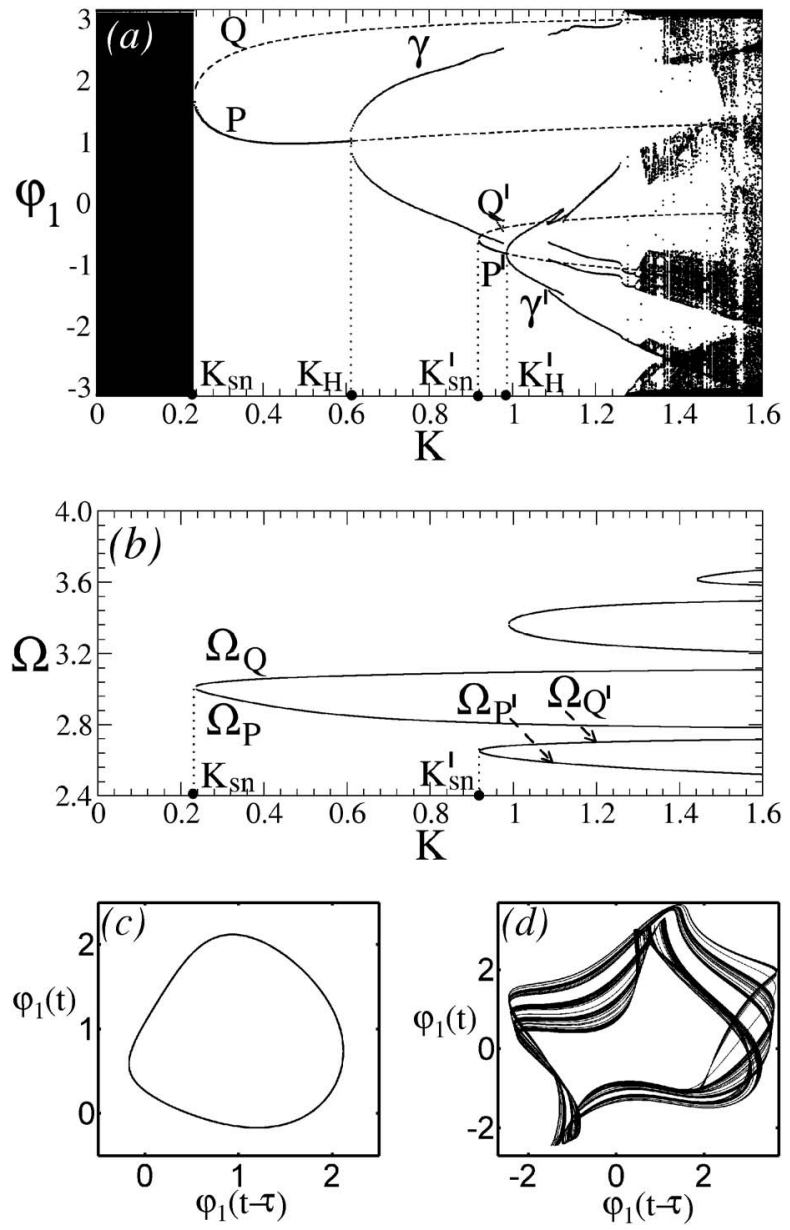

FIG. 2. (a) Dynamical regimes of the phase difference $\varphi_{1}$ in system (4) versus coupling strength $K$. When the coupling strength increases, system (4) undergoes a sequence of bifurcation transitions from desynchronization via stable phase-locked and periodically modulated synchronized states to chaotic phase synchronization and, finally, to desynchronization again. Stable and unstable phase-locked states are depicted by solid and dashed curves, respectively. For oscillatory regimes (limit cycles and chaotic attractors) only local maxima and minima of the time courses of trajectories are plotted. $K_{S N}, K_{S N}^{\prime}$ and $K_{H}, K_{H}^{\prime}$ are the bifurcation values of $K$ of the saddle-node $(\mathrm{SN})$ and Hopf $(H)$ bifurcations. (b) Corresponding frequencies $\Omega$ of the phase-locked states versus $K$. Examples of a limit-cycle oscillation for $K=0.8$ and a chaotic attractor for $K$ $=1.34$ are shown in the $\left[\varphi_{1}(t-\tau), \varphi_{1}(t)\right]$-phase space in (c) and $(\mathrm{d})$, respectively. Parameters $\Delta_{1}=0.2, \Delta_{2}=3.0$, and $\tau=4.0$.

parameter bifurcation diagram of system (4) is presented for $\varphi_{1}$ versus $K$. When the coupling parameter $K$ increases, a stable phase-locked state $P$ and a saddle phase-locked state $Q$ are born in a saddle-node bifurcation at $K=K_{S N}$, where $K_{S N} \approx 0.235$ in Fig. 2(a). In a phase-locked state, the phase difference is constant and the mean phase grows with a mean frequency $\Omega$ :

$$
\varphi_{1}(t)=\varphi_{1}^{*}, \quad \varphi_{2}(t)=\Omega t+\text { const } .
$$

We also call the phase-locked states "fixed points". Inserting expressions (5) into Eq. (4) one obtains that the mean fre- 
quency $\Omega$ and the phase shift $\varphi_{1}^{*}$ of the phase-locked states (5) obey

$$
\begin{aligned}
-\Omega+\Delta_{2} \pm \frac{K}{2} \sin \Omega \tau \sqrt{1-\frac{\Delta_{1}^{2}}{K^{2} \cos ^{2}(\Omega \tau)}}=0, \\
\varphi_{1}^{*}=\left\{\begin{array}{l}
\arcsin \left(\frac{\Delta_{1}}{K \cos (\Omega \tau)}\right), \\
\pi-\arcsin \left(\frac{\Delta_{1}}{K \cos (\Omega \tau)}\right),
\end{array}\right.
\end{aligned}
$$

where the first and the second values of $\varphi_{1}^{*}$ in Eq. (7) correspond to the signs "+" and "-" in Eq. (6), respectively.

The birth of the phase-locked states $P$ and $Q$ is induced by the emergence of a pair of frequencies $\Omega_{P}$ and $\Omega_{Q}$, solutions of Eq. (6), see Fig. 2(b). According to Eq. (5), $\Omega_{P}$ and $\Omega_{Q}$ are the mean frequencies of the two oscillators (1) in the phase-locked states $P$ and $Q$, respectively. The fixed point $P$ is stable for $K \in\left(K_{S N}, K_{H}\right)$, where $K_{H} \approx 0.61$ is the moment of its Hopf bifurcation [Fig. 2(a)]. At $K=K_{H}$ the fixed point $P$ loses its stability and a stable limit cycle $\gamma$ emerges [Figs. 2(a) and 2(c)]. After the bifurcation, the phase difference $\varphi_{1}(t)$ of trajectories attracted by $\gamma$ is not constant and exhibits periodic oscillations. It is still bounded and, thus, a regime of periodically modulated phase synchronization is established in system (1).

The number of different solutions $\Omega$ of Eq. (6) grows as the parameter $\mathrm{K}$ increases. For example, after the first pair of frequencies $\Omega_{P}$ an $\Omega_{Q}$ corresponding to the phase-locked states $P$ and $Q$, the second pair of solutions $\Omega_{P^{\prime}}$ and $\Omega_{Q^{\prime}}$ of Eq. (6) emerges at $K=K_{s n}^{\prime} \approx 0.92$ giving birth to a new stable phase-locked state $P^{\prime}$ and a saddle phase-locked state $Q^{\prime}$ of system (1) [Fig. 2(a) and 2(b)]. The fixed point $P^{\prime}$ is stable for $K \in\left(K_{S N}^{\prime}, K_{H}^{\prime}\right)$ and loses its stability with increasing $K$ at $K=K_{H}^{\prime} \approx 0.985$ via a Hopf bifurcation. In this bifurcation a stable limit cycle $\gamma^{\prime}$ emerges [Fig. 2(a)]. With a further increase of the coupling strength new pairs of stable and saddle phase-locked states appear, corresponding to the emergence of new solutions $\Omega$ of Eq. (6). The stable phase-locked states, exist in only narrow intervals of the parameter $K$ values, bifurcate via supercritical Hopf bifurcations, loose their stability, and give birth to stable limit cycles.

At larger values of $K$ periodic motion in system (4) turns into chaotic motion [Fig. 2(a) and 2(d)]. The phase difference $\varphi_{1}$ still remains bounded, which indicates an emergence of a chaotic phase synchronization according to definition in Ref. [50]. Further, if $K$ reaches some critical value $K=K_{\mathrm{cr}}$, an attractor of chaotic phase synchronization undergoes a crisis and system (1) returns to a desynchronized state, where the phase difference $\varphi_{1}(t)$ displays unbounded rotations [Fig. 2(a)].

The stable phase-locked states of system (1) may coexist, which leads to multistability. This takes place when the next stable phase-locked state, say $P^{\prime}$, is born before the previously emerging phase-locked state, say $P$, loses its stability via a Hopf bifurcation. An example of two coexisting stable phase-locked states is illustrated in Fig. 3. Here, both states $P$ and $P^{\prime}$ are in-phase locked states with $\varphi_{1}^{*}=0$ with different

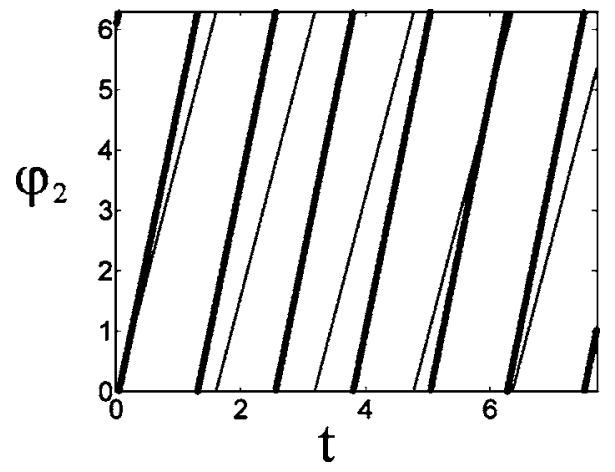

FIG. 3. Coexistence of two stable in-phase-locked states $P$ and $P^{\prime}$ with the same phase difference $\varphi_{1}^{*}=0$, but with different frequencies $\Omega_{P}=5.12$ and $\Omega_{P^{\prime}}=3.96$, respectively. Time courses of the mean phase $\varphi_{2}$ are shown for stable states $P$ (bold line) and $P^{\prime}$ (thin line). Parameters $\Delta_{1}=0.0, \Delta_{2}=4.5, \tau=1.4$, and $K=1.6$.

frequencies $\Omega_{P}=5.12$ and $\Omega_{P^{\prime}}=3.96$, respectively. The both states are realized in system (1) for the same parameter values but for different initial conditions.

The multistability phenomenon emerges in system (1) not only between stable phase-locked states, but also between other synchronous and desynchronous states. For instance, in Fig. 2(a) one can see that the stable fixed point $P^{\prime}$ can coexist with the stable limit cycle $\gamma$ and also two stable limit cycles $\gamma$, and $\gamma^{\prime}$ can coexist. For some other set of parameter values, also a stable desynchronous dynamics, where the phase difference $\varphi_{1}$ exhibits unbounded rotations, and a stable synchronous state with bounded $\varphi_{1}$ can coexist in system (1), as illustrated in Fig. 4. A region in the $(\tau, K)$-parameter plain, where a stable synchronous state coexists with a stable desynchronous limit cycle is depicted in Fig. 4(a). The lowest curve corresponds to the moment of the birth of a stable phase-locked state $P$. As $K$ increases this point then bifurcates into a stable synchronous limit cycle $\gamma$ via a Hopf bifurcation (middle curve). For parameter values of the gray region between "phase-locking" and "desynchronization" curves, system (1) displays a stable synchronized motion, where the phase difference $\varphi_{1}$ is bounded. On the other hand, the hatched region corresponds to parameter values, where a stable desynchronous limit cycle $\mu$ exists, characterized by unbounded rotations of the phase difference $\varphi_{1}$ on the torus. An example of the stable fixed point $P$ coexisting with the stable desynchronous limit cycle $\mu$ is illustrated in Fig. 4(b) for parameter values indicated by point $A$ in Fig. 4(a). Depending on the initial conditions, system (1) displays one or the other stable dynamics.

Below we study the responses of system (1) on stimulation (2) in the following four dynamical regimes: (i) a single stable phase-locked state, (ii) stable, periodically modulated synchronization, (iii) bistability of two phase-locked states, and (iv) a regime of bistability of a phase-locked state and a desynchronous limit cycle. We explore the intertrial clustering of the oscillators' responses emerging during in- and post-stimulus transients for a stimulated phase-locked state. Optimal parameter values are detected, where recovery time (i.e., the duration) of the post-stimulus transient gets maximal. We also uncover the mechanism of such a maximal 

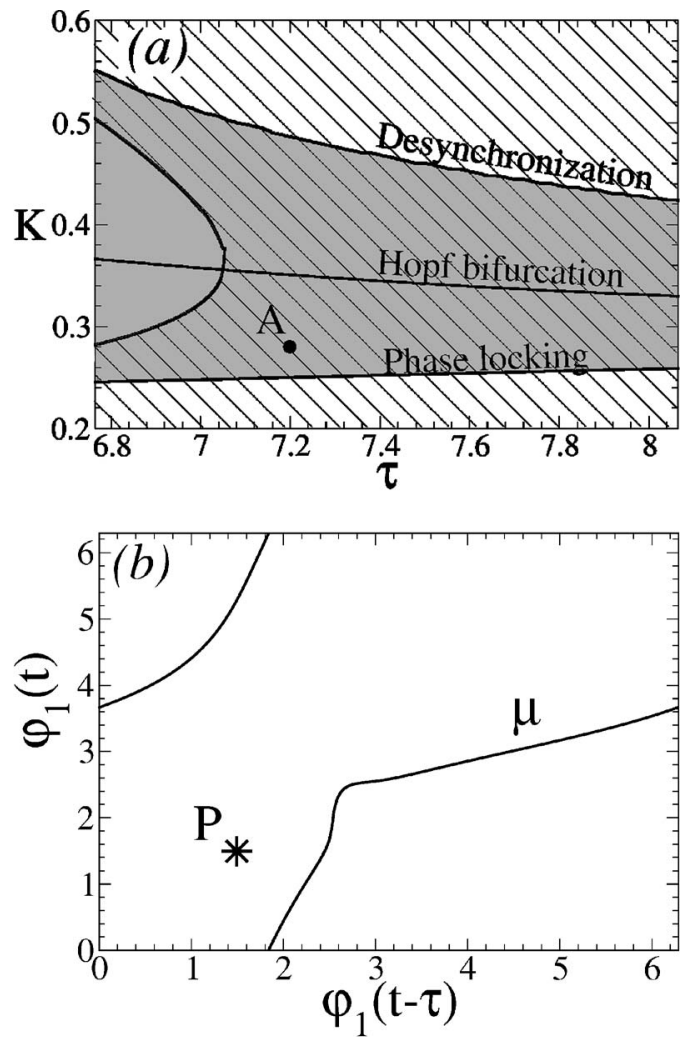

FIG. 4. Coexistence of stable synchronized and desynchronized states of system (1). (a) Bifurcation diagram in the $(\tau, K)$-parameter plain. The gray and hatched regions correspond to the existence of stable synchronized and desynchronized states, respectively. (b) Example of the coexistence of the stable desynchronous limit cycle $\mu$ and the stable phase-locked state $P$ (asterisk) for parameters $K$ $=0.28$ and $\tau=7.2$ indicated by point $A$ in (a). Other parameters $\Delta_{1}$ $=0.2$ and $\Delta_{2}=0.1$.

transient. For a stimulated periodically modulated synchronized state we analyze the post-stimulus transient and compare it with that of a phase-locked state. Finally, for stimulated multistable regimes we provide an evidence of stimulus-induced switching between synchronized and desynchronized motions.

\section{DYNAMICS DURING STIMULATION}

In the framework of a statistical approach to transient stimulus-locked dynamics [14-18], (see also the Appendix) we consider the following stimulation protocol: A series of $N$ identical stimuli (2) are administered to system (1) consecutively one stimulus after the other. Each stimulus acts only during a short time interval of duration $T_{\mathrm{st}}$. The length of the interstimulus intervals is stochastically varied from one stimulus to another and is large enough to let the system return to its own dynamical regime, before the next stimulus is applied. Around each stimulus a time window of the length $T_{w}$ is attached, in which the evaluation of the trajectories of system (1) is performed across trials. During a post-stimulus transient, when stimulation is off $(X=0)$, system (1) relaxes towards its stable state displaying different kinds of re- sponses to the stimulation. These post-stimulus responses are the subject of our study. The goal of our approach is to detect stereotypical features of the (transient) dynamics of the phases $\psi_{1}$ and $\psi_{2}$, the phase difference $\varphi_{1}$, and the mean phase $\varphi_{2}$ in an ensemble of post-stimulus responses.

Let system (1) be first stimulated in a regime, with only one stable phase-locked state. For this, the values of the parameters of system (1) are chosen such as in Fig. 2(a), where a stable fixed point $P$ and a saddle point $Q$ exist. Generic system responses extracted from $N=300$ stimulation trials with a stochastic phase response analysis [14-16,18] are presented in Fig. 5.

We use cross-trial distributions of the variables $\psi_{1,2}$ and $\varphi_{1,2}$ [Figs. 5(a), 5(c), 5(e), and 5(g)] and the corresponding stimulus-locked indices (see the Appendix) [Figs. 5(b), 5(d), 5(f), and 5(h)] to study the stimulus-locked dynamics. Before stimulus onset the phases $\psi_{1}$ and $\psi_{2}$ are uniformly distributed in $[0,2 \pi)$ since stimuli are administered randomly. Accordingly, both resetting indices $\rho_{j}$ and clustering indices $\alpha_{j}, j$ $=1,2$, are close to 0 . A stimulus of the form $I \cos \left(\psi_{j}-\theta_{j}\right)$ [see Eq. (2)] of sufficient strength $(I \gg K)$ and duration rapidly resets the phase of the $j$ th oscillator to the phase $\psi_{\text {res }}$ $+\theta_{j}[14-16,18][$ see also Eq. (10)]. Hence, at stimulus offset the resetting indices $\rho_{1}$ and $\rho_{2}$ are close to 1 , which indicates a complete reset of the phases. The phases $\psi_{1}$ and $\psi_{2}$ are reset to particular values $\psi_{1}\left(t_{E}\right)$ and $\psi_{2}\left(t_{E}\right)$ irrespective of their initial values at stimulus onset, where $t_{E}$ denotes stimulus offset. Note, $\psi_{1}\left(t_{E}\right) \neq \psi_{2}\left(t_{E}\right)$ due to the nonvanishing shift term $\theta_{2}$ of the second stimulus [Eq. (10)].

Before stimulus onset the two oscillators are strongly synchronized with a nonvanishing phase difference $\varphi_{1}$ [Fig. 5(e)]. This stereotypical phase relationship between the two oscillators shows up as a dirac-type pre-stimulus distribution of $\varphi_{1}$ and, thus, the synchronization index $\sigma_{1}$ is close to 1 , where the value of the phase difference $\varphi_{1}$ remains fixed within the entire prestimulus interval. The stimuli reset the oscillators in a way that their phase difference is set to a value different to that of the pre-stimulus range [Fig. 5(e)]. This processes is reflected by a quick decrease and subsequent reincrease of the synchronization index $\sigma_{1}$, which occurs directly after stimulus onset [Fig. 5(f)].

After stimulus offset the oscillators relax back to their stable prestimulus phase difference. This is achieved in two different ways, where one of the oscillators speeds up relative to the other one, respectively [Figs. 5(a) and 5(c)]. Accordingly, the phase difference either increases $(\bmod 2 \pi)$ or decreases [Fig. 5(e)]. This two-branch type resynchronization process of $\varphi_{1}$ shows up as a transient epoch of desynchronization, where the synchronization index $\sigma_{1}$ of $\varphi_{1}$ decreases [Fig. 5(f)]. The clustering index $\delta$ of the phase difference $\varphi_{1}$ gets maximal when the two branches are in antiphase position. A further consequence of the two branches of transient trajectories is the emergence of a response clustering of both oscillators $\psi_{1}$ and $\psi_{2}$ : After stimulus offset the oscillators restart from the reset state [Figs. 5(a) and 5(c)], the resetting indices $\rho_{1}$ and $\rho_{2}$ decrease, whereas the clustering indices $\alpha_{1}$ and $\alpha_{2}$ increase above the prestimulus level, indicating an anti-phase response clustering [Figs. 5(b) and 5(d)]. Put otherwise, after an initial reset each os- 

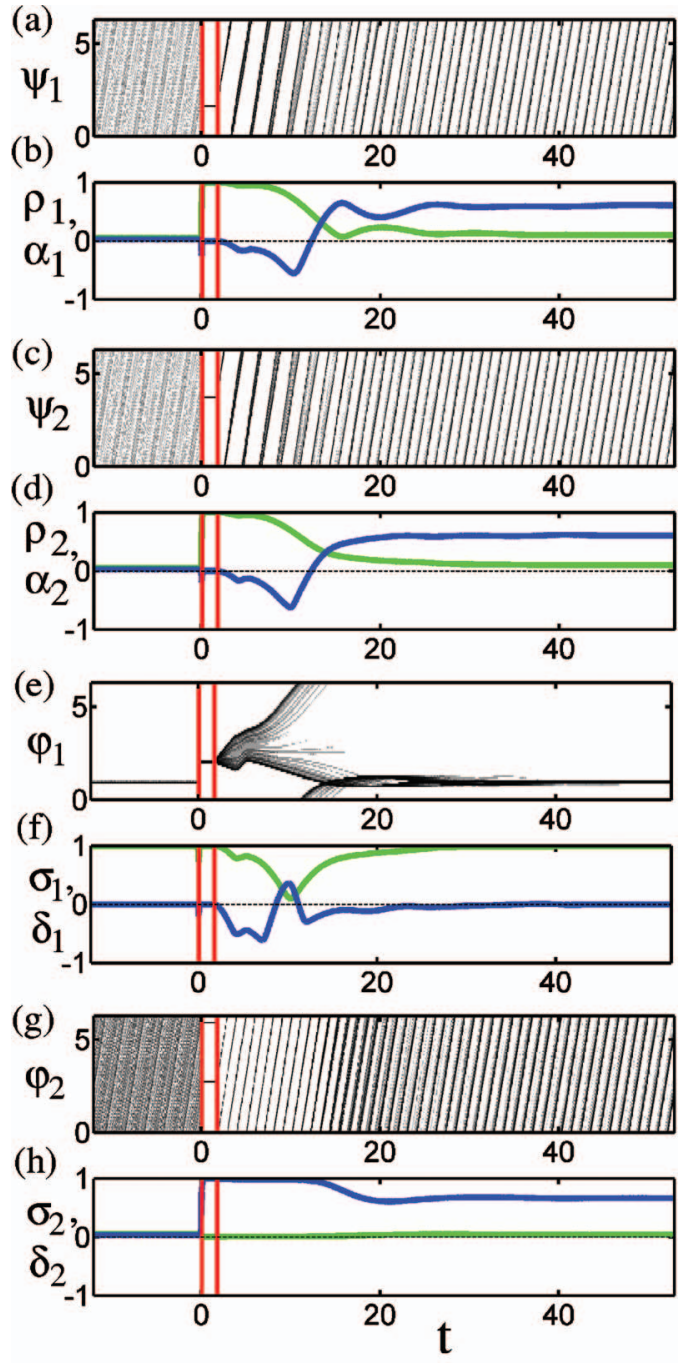

FIG. 5. (Color) Stimulus-locked responses of system (1) stimulated in the phase-locked regime with signal (2). Cross-trial distributions of the phases $\psi_{1}, \psi_{2}$, the phase difference $\varphi_{1}$, and the mean phase $\varphi_{2}$ are shown in (a), (c), (e), and (g), respectively, where the density of trajectories is encoded by a gray scale (minimum is white, maximum is black). Onset (at time $t=0$ ) and offset of the stimuli are indicated by vertical red lines. Characteristic features of the stimulus-locked dynamics as revealed by cross-trail indices (see the Appendix and Refs. [14-16,18]): The resetting indices $\rho_{1}$ and $\rho_{2}$ and the clustering indices $\alpha_{1}$ and $\alpha_{2}$ of the phases $\psi_{1}$ and $\psi_{2}$ are shown in (b) and (d); the synchronisation indices $\sigma_{1}$ and $\sigma_{2}$ and the clustering indices $\delta_{1}$ and $\delta_{2}$ of the phase difference $\varphi_{1}$ and the mean phase $\varphi_{2}$ are shown in (f) and (h). The resetting/synchronization indices (green curves) and the clustering indices (blue curves) detect whether the corresponding cross-trial distribution has one pronounced peak or two pronounced antiphase peaks. Number of stimuli $N=300$. Parameters: $\Delta_{1}=0.2, \Delta_{2}=3.0, K=0.4, \tau=4.0$, and $\theta=2.075$.

cillator displays two anti-phase types of responses across trials. This corresponds to the cross-trail distribution of $\psi_{j}$ having two antiphase peaks [Figs. 5(a) and 5(c)] (see the Appendix).

Additionally, there is a clustering process of the mean phase $\varphi_{2}$ [Figs. $5(\mathrm{~g})$ and $\left.5(\mathrm{~h})\right]$. As one can see in Fig. 5(g), this clustering process occurs already during stimulation, directly after stimulus onset, where two antiphase clusters of the variable $\varphi_{2}$ are formed. During the post-stimulus transient these clusters get slightly smeared. Correspondingly, the prestimulus resetting and clustering indices $\sigma_{2}$ and $\delta_{2}$ of the mean phase are close to zero because of the randomized stimulus administration [Fig. $5(\mathrm{~g})]$. The clustering index $\delta_{2}$ first quickly increases after stimulus onset, and then, after a transient slight decrease, finally remains above the prestimulus level, as soon as the resynchronization is achieved [Figs. 5(e) and 5(f)]. The combination of increased $\delta_{2}$ and almost zero $\sigma_{2}$ is indicative of two antiphase peaks of the cross-trial distributions of $\varphi_{2}$ [Fig. 5(g), see also Refs. [14-16,18]]. The numbers of trials in each cluster (i.e., peak of the bimodal cross-trial distribution of $\varphi_{2}$ ) are 149 and 151, out of a total $N=300$ stimulation trials. In contrast, in [Figs. 5(a), 5(c), and $5(\mathrm{e})$ the phase variables $\psi_{1}$ and $\psi_{2}$, and the phase difference $\varphi_{1}$ simultaneously split into two antiphase clusters with 140 and 160 trials, respectively. Therefore, the clustering of the variable $\varphi_{2}$ does not correspond to the post-stimulus clustering of the other variables, as we will also show below.

We consider the formation of the response clusters in more detail. We show that the response clustering of the phase difference $\varphi_{1}$ plays an important role in the formation of the response clustering of the phases $\psi_{1}$ and $\psi_{2}$. For this, we first investigate the dynamics of system (1), (2) during stimulation, i.e., in-stimulus dynamics of Eqs. (1), (2). Second, we study the post-stimulus transient of system (1).

In-stimulus clustering. If a strong stimulus (2) $\left(I \gg \omega_{j}, I \gg K\right)$ is applied to system (1), a phase reset occurs (Fig. 5). This means that in a short transient after stimulus onset, stimulation shifts both phase oscillators (1) to a stimulus-locked state, where both phases become constant. Considering system (1), (2) in variables (3) during stimulation, and neglecting the coupling (because $K \ll I$ and $\omega_{j} \ll I$ ), we obtain a new system:

$$
\begin{aligned}
& \dot{\tilde{\varphi}}_{1}(t)=-2 I \sin \left(\frac{\tilde{\varphi}_{1}(t)-\theta}{2}\right) \sin \left(\tilde{\varphi}_{2}(t)-\frac{\theta}{2}\right), \\
& \dot{\tilde{\varphi}}_{2}(t)=I \cos \left(\frac{\widetilde{\varphi}_{1}(t)-\theta}{2}\right) \cos \left(\widetilde{\varphi}_{2}(t)-\frac{\theta}{2}\right),
\end{aligned}
$$

where the variables $\tilde{\varphi}_{i}$ approximate the variables $\varphi_{i}$ defined by Eq. (3). For strong enough stimulus (2), system (8) governs the in-stimulus dynamics of Eqs. (1), (2). It has the following stationary solutions $\left[\widetilde{\varphi}_{1}(t), \widetilde{\varphi}_{2}(t)\right]=\left(\widetilde{\varphi}_{1}^{*}, \widetilde{\varphi}_{2}^{*}\right)$ :

$$
\tilde{\varphi}_{1}^{*}=\theta+\pi n, \quad \widetilde{\varphi}_{2}^{*}=\frac{\theta}{2}+\frac{\pi}{2} m, \quad n, m \in \mathbb{Z} .
$$

Since system (8) is $4 \pi$ periodic in $\tilde{\varphi}_{1}$ and $2 \pi$ periodic in $\tilde{\varphi}_{2}$, it has eight different fixed points (9) in the $[0 ; 4 \pi)$ $\times[0 ; 2 \pi)$-phase space: two stable nodes $A=\left(\theta, \theta / 2+\frac{\pi}{2}\right)$ and $B=\left(\theta+2 \pi, \theta / 2+\frac{3 \pi}{2}\right)$ with eigenvalues $\lambda_{1,2}=-I$, two unstable nodes $\left(\theta, \theta / 2+\frac{3 \pi}{2}\right)$ and $\left(\theta+2 \pi, \theta / 2+\frac{5 \pi}{2}\right)$ with eigenvalues $\lambda_{1,2}=I$, and four saddles $(\theta+\pi+2 \pi n, \theta / 2+\pi m)$ with $n, m$ $=0,1$ and eigenvalues $\lambda_{1}=I, \lambda_{2}=-I$. In Fig. 6 the phase portrait of system (8) with fixed points (9) and with stable and 


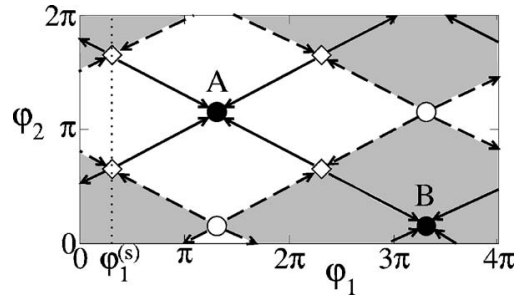

FIG. 6. Fixed points of system (8) induced by stimulation. Black filled circles (points A and B) indicate stable nodes, empty circles indicate unstable nodes, and diamonds indicate saddles. Dashed and solid lines depict stable and unstable manifolds of the saddles, respectively. Basins of attraction of the stable fixed points $A$ and $B$ are indicated by white and gray areas, respectively. The vertical dotted line shows the $\varphi_{1}$-coordinate $\varphi_{1}^{(s)}$ of the stable phase-locked state of system (1) and corresponds to the initial conditions of the system (4) at stimulus onset. Parameters: $\theta=4.11, \Delta_{1}=0.2, \Delta_{2}=3.0, K$ $=0.4$, and $\tau=4.0$.

unstable manifolds of the saddles is shown for $\theta=4.11$. The net formed by the stable manifolds of the saddle points (dashed lines with arrows) divides the whole $\left(\widetilde{\varphi}_{1}, \widetilde{\varphi}_{2}\right)$-phase plain into basins of attraction of the stable fixed points $A$ (white regions) and $B$ (gray regions). Therefore, for $t \geq 0$ a generic trajectory $\left[\widetilde{\varphi}_{1}(t), \widetilde{\varphi}_{2}(t)\right]$ of system (8) will be attracted by either the stable fixed point $A$ or $B$, depending on the basin of attraction in which the trajectory starts, as given by initial conditions $\left[\widetilde{\varphi}_{1}(0), \widetilde{\varphi}_{2}(0)\right]$. During stimulation the phases $\psi_{i}$ of system (1), (2) approach a stationary reset state: Both phases become approximately constant (Fig. 5). The corresponding coordinates $\left(\varphi_{1}, \varphi_{2}\right)$ in the reset state are approximated by the coordinates of the stable fixed points $A$ and $B$ of the approximative system (8).

The initial conditions $\left[\widetilde{\varphi}_{1}(0), \widetilde{\varphi}_{2}(0)\right]$ of system (8) equal the values of the variables $\left[\varphi_{1}(t), \varphi_{2}(t)\right]$ of system (4) at stimulus onset. For the stimulated phase-locked states (5) the initial conditions $\left[\widetilde{\varphi}_{1}(0), \widetilde{\varphi}_{2}(0)\right]$ attain the following values: In all trials the phase difference reads $\widetilde{\varphi}_{1}(0)=\varphi_{1}^{(s)}$, whereas the mean phase $\widetilde{\varphi}_{2}(0)$ fills the whole segment $[0 ; 2 \pi)$ across trials. The latter fact is because of the linear growth of the mean phase $\varphi_{2}$ according to Eq. (5) and randomly varying interstimulus time intervals. Here, $\varphi_{1}^{(s)}$ denotes the $\varphi_{1}$ coordinate of the stable fixed point of system (4), for example, point $P$ shown in Fig. 2(a). Hence, summarizing the initial conditions of Eq. (8) over all stimulation trials, one can represent them as a vertical segment $\varphi_{1}^{(s)} \times[0 ; 2 \pi)$ in the phase space in Fig. 6 [dotted line]. The fixed points $A$ and $B$ of Eq. (8) are located on different sides with respect to the initial segment above (when consider a lift of the torus $[0 ; 2 \pi$ ) $\times[0 ; 4 \pi)$ to $\left.\mathbb{R}^{2}\right)$, i.e., on different sides with respect to the initial phase shift $\widetilde{\varphi}_{1}(0)=\varphi_{1}^{(s)}$. Therefore, during in-stimulus transient on the way towards the fixed point $A(B)$, the phase difference $\widetilde{\varphi}_{1}(t)$ increases (decreases). Furthermore, during the in-stimulus transient $\widetilde{\varphi}_{2}$ approaches either $A$ or $B$, accordingly. Such a splitting of the trajectories of system (8) between the basins of attraction of the reset states $A$ and $B$ is the mechanism which causes the in-stimulus clustering in system (1), (2), see Fig. 5.

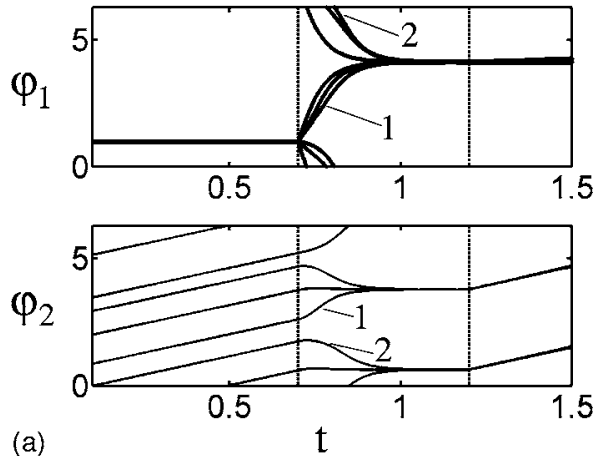

(a)
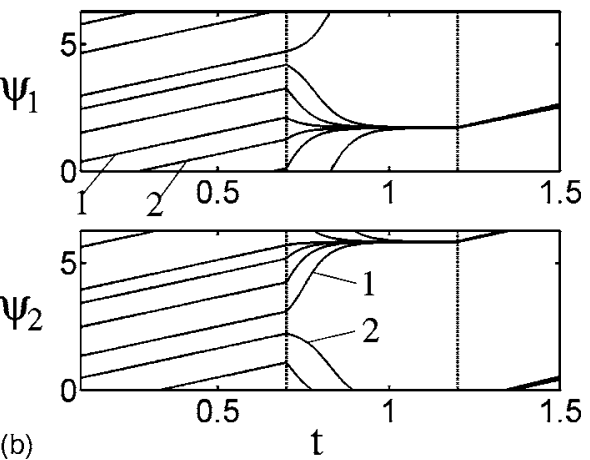

FIG. 7. In-stimulus clustering illustrated for a few trajectories of the stimulated system (1), (2) with different initial conditions. Variables $\varphi_{1}(t), \varphi_{2}(t), \psi_{1}(t)$, and $\psi_{2}(t)$ are separately shown on each plot. In-stimulus clustering is induced by attraction to different reset states $A$ or $B$ (Fig. 6). Two selected trajectories belonging to different clusters are denoted by numbers 1 and 2 . The on- and off-sets of stimulation are indicated by vertical dashed lines. $\theta=4.11$ and the other parameters are as in Fig. 5.

The clustering phenomenon for the in-stimulus transient of system (1), (2) is additionally illustrated in Fig. 7, where transients towards $A$ and $B$ are shown for a few selected trajectories of Eqs. (1), (2). Trajectories such as No. 1 are attracted by $A$ and their coordinate $\varphi_{1}$ increases, whereas trajectories such as No. 2 are attracted by $B$ and $\varphi_{1}$ decays. Note, the two reset states given by the fixed points $A$ and $B$ of Eq. (8) are indistinguishable when considering cross-trial distributions of the variables $\psi_{i}, j=1,2$, and $\varphi_{1}$ taken modulo $2 \pi$ (Fig. 5):

$$
\begin{gathered}
\left(\psi_{1}, \psi_{2}\right) \approx(\pi / 2, \theta+\pi / 2) \text { corresponds to } \\
\left(\varphi_{1}, \varphi_{2}\right) \approx(\theta, \theta / 2+\pi / 2)(A) \text { and } \\
\left(\varphi_{1}, \varphi_{2}\right) \approx(\theta, \theta / 2+3 \pi / 2)(B) .
\end{gathered}
$$

The trajectories' twofold convergence to the reset states $A$ and $B$ constitutes the in-stimulus response clustering of the mean phase $\varphi_{2}$ [Fig. $\left.5(\mathrm{~g})\right]$. Two trajectories belong to different clusters if they are attracted by different reset attractors $A$ or $B$, see Figs. 6 and 7. Consider a clustering measure $H$ defined as the relative number of trajectories of system (1), (2) within the smallest cluster during the in-stimulus transient. Evidently, $H \in[0 ; 0.5]$. This clustering measure can be calculated as a function of $\theta$ if one compares the numbers of 


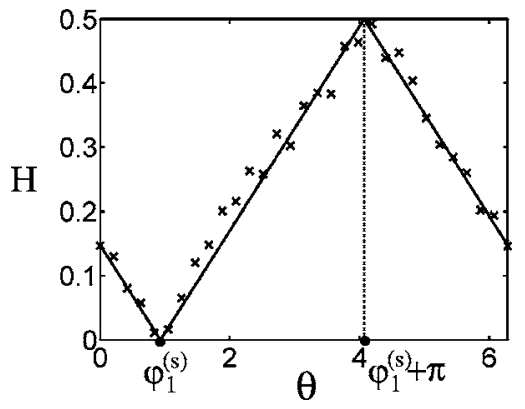

FIG. 8. Clustering measure $H$, i.e., the relative number of trajectories in the smallest cluster during in-stimulus clustering of system (1), (2) versus stimulation phase shift $\theta$. Solid line shows the theoretical estimation according to Eq. (11) and crosses show results of a series of numerical simulations of system (1), (2) for different values of $\theta \cdot \varphi_{1}^{(s)}$ denotes the $\varphi_{1}$ coordinate of the stable fixed point of system (4). Parameters $\Delta_{1}=0.2, \Delta_{2}=3.0, K=0.4, \tau$ $=4.0$, and $N=1300$.

trajectories $\left\{\left(\varphi_{1}(t), \varphi_{2}(t)\right)\right\}$ of system (1), (2) belonging to the basins of attraction of points $A$ and $B$ at the stimulus onset time moments. For the case, where the mean phase $\varphi_{2}(t)$ fills the segment $[0 ; 2 \pi)$ uniformly over stimulation trials, the clustering measure $H$ is the length of fractions of the segment $\varphi_{1}^{(s)} \times[0 ; 2 \pi)$ that belong to the basin of attraction of $A$ or $B$ (Fig. 6). Under this condition, the following formula gives an approximation of the clustering measure $H$ :

$$
H(\theta)=-\left|\frac{\left[\left(\varphi_{1}^{(s)}-\theta\right) \bmod 2 \pi\right]-\pi}{2 \pi}\right|+\frac{1}{2} .
$$

The graph of the clustering measure $H$ given by Eq. (11) (solid line in Fig. 8) is in good agreement with the values of $H$ obtained by simulating system (1), (2), (crosses in Fig. 8). There is an optimal value of the parameter $\theta=\theta_{\mathrm{cl}}=\left(\varphi_{1}^{(s)}\right.$ $+\pi) \bmod 2 \pi$, where the in-stimulus clustering is maximal, i.e., the two emerging clusters are of equal size. This situation occurs if the $\varphi_{1}$ coordinates of the saddle fixed points of system (8), which are $\theta+\pi+2 \pi n, n=0,1$, coincide with the $\varphi_{1}$ coordinate $\varphi_{1}^{(s)}$ of the stable fixed point $P$. In this case equally long fractions of the segment $\varphi_{1}^{(s)} \times[0 ; 2 \pi)$ belong to the basins of attractors $A$ and $B$, respectively, as shown in Fig. 6.

The in-stimulus clustering determines the post-stimulus clustering of the mean phase $\varphi_{2}$ (Fig. 5), where the clusters of trajectories emerging during stimulation are preserved during the post-stimulus transient. For short and weak stimulation, when the phases are reset for a time smaller than the delay $\tau$, the in-stimulus clustering of the phase difference $\varphi_{1}$ also plays an important role for the post-stimulus clustering of the phases $\psi_{1}$ and $\psi_{2}$. However, in case of a strong and long enough stimulus, the in-stimulus clustering is of minor importance for the post-stimulus clustering of the phase variables $\psi_{1}$ and $\psi_{2}$. Below we explore in which way the global structure of the phase space of system (4) influences the poststimulus clustering in system (1), (2).

\section{POST-STIMULUS TRANSIENT TO A SYNCHRONIZED STATE}

Stimuli of sufficient strength and duration shift the trajectories of system (1), (2) very close to the reset state (10), where they remain till the end of the stimulation. After stimulus offset system (1) returns back to its own stable state. In this section we study the post-stimulus transients of system (1) occurring in the phase-locked and periodically modulated synchronized regimes.

\section{A. Stimulation of phase-locked states}

Let us, as before, consider the case, where system (1) has a singe stable phase-locked state $P$. After stimulus offset, trajectories relax from the stimulus-induced reset state (10) towards the phase-locked state $P$. An example of such a transient is shown in the cross-trail distributions in Fig. 5. One of the important characteristics here is the transient time $T_{\mathrm{tr}}$. We define the transient time $T_{\mathrm{tr}}$ as the time it takes a trajectory after stimulus offset to permanently enter into an $\varepsilon$ vicinity of the stable phase-locked state, averaged over the ensemble of $N$ stimuli. We study how $T_{\text {tr }}$ depends on the stimulation phase shift $\theta$ for long and strong enough stimuli.

An example of the transient time $T_{\mathrm{tr}}$ calculated for the stimulated system (1), (2) without delay, i.e., for $\tau=0$ in Eq. (1), is shown in Fig. 9(a) versus $\theta$. One can see that there are two critical values of the stimulation phase shift $\theta_{\max }$ and $\theta_{\text {min }}$, where the transient time attains its maximum and minimum, respectively. Moreover, the stimulus length $T_{\text {st }}$ has a minor influence on the transient time $T_{\mathrm{tr}}$, as demonstrated for $T_{\text {st }}=0.75,2.25$, and 4.75 in Fig. 9(a), where the three curves for the different stimulation times coincide. The critical values $\theta_{\min }$ and $\theta_{\max }$ correspond to the stable $P$ and the unstable $Q$ fixed points of system (4) without delay with $\varphi_{1}$ coordinates $\varphi_{1}^{(s)} \approx 0.52$ and $\varphi_{1}^{(u)} \approx 2.62$, respectively. Evidently, in order to obtain the longest post-stimulus transient in system (1), (2) without delay, the stimulation has to shift the trajectories towards the unstable fixed point of Eq. (4). This can be done by a proper choice of the stimulus phase shift $\theta$, namely, $\theta$ has to be taken equal to the $\varphi_{1}$ coordinate $\varphi_{1}^{(u)}$ of the unstable fixed point $Q$.

The situation is different if the stimulated system has a significant time delay. This is illustrated in Fig. 9(b), where the transient time $T_{\mathrm{tr}}$ is plotted versus $\theta$ for the delay $\tau$ $=4.0$ and for the same three values of the stimulus length as above $T_{\mathrm{st}}=0.75,2.25$, and 4.75 . For parameter values as in Fig. 9(b), system (4) has two fixed points: stable $P$ and saddle $Q$ with coordinates $\varphi_{1}^{(s)} \approx 0.997$ and $\varphi_{1}^{(u)} \approx 2.583$, respectively, see Fig. 2(a). If the stimulation time $T_{\text {st }}$ is relatively small (in comparison with the delay $\tau$ ), the transient time $T_{\text {tr }}$ still has a maximum, which is not directly located at the coordinate $\varphi_{1}^{(u)}$ of $Q$ but close to it [maximum 1 in Fig. 9(b)]. The dependence of the transient time on the phase shift $\theta$ undergoes a significant change when $T_{\text {st }}$ increases: The maximum of $T_{\mathrm{tr}}$ is shifted to the left [maximum 2 in Fig. 9 (b)] and finally saturates at a critical point $\theta_{\max }$, located between the stable fixed point $P$ and the unstable fixed point $Q$ [Fig. 9(b)]. Therefore, in order to obtain the maximal poststimulus transient time in the system with delay (1) stimu- 

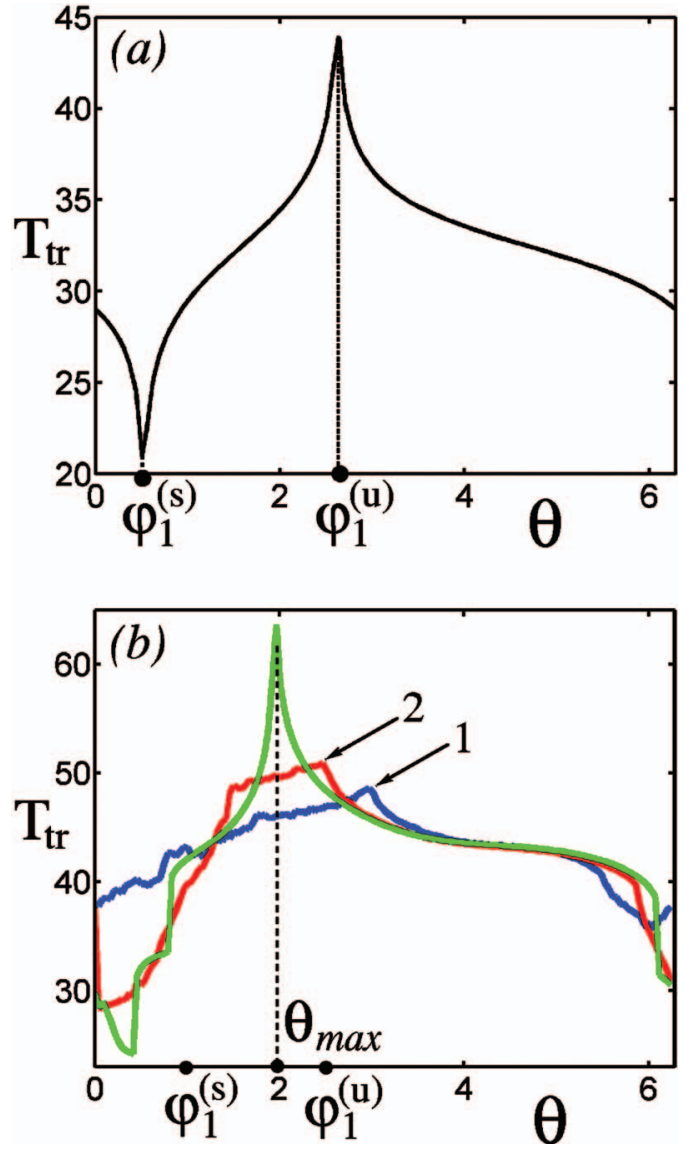

FIG. 9. (Color) Transient time $T_{t r}$ for the post-stimulus relaxation of system (1), (2), necessary to reestablish its stable phaselocked state, versus stimulation phase shift $\theta$. Delay: (a) $\tau=0$ and (b) $\tau=4.0$. In both plots three graphs are shown for different stimulus lengths $T_{\mathrm{st}}=0.75$ (blue), $T_{\mathrm{st}}=2.25$ (red), and $T_{\mathrm{st}}=4.75$ (green). Coinciding curves in (a) are indicated by a black curve. Maxima of blue and red curves in (b) are indicated by " 1 " and " 2 ," respectively. $\varphi_{1}^{(s)}$ and $\varphi_{1}^{(u)}$ indicate the $\varphi_{1}$ coordinates of the stable and unstable fixed points of system (4), respectively. Number of stimuli $N=200$ and $\varepsilon=0.05$. Parameters: $\Delta_{1}=0.2, \Delta_{2}=3.0, K=0.4, I=30.0$.

lated with signal (2) of sufficient duration, one has to stimulate the system into a neighborhood of an optimal point $\theta_{\max }$ which is different from the $\varphi_{1}$-coordinate of the unstable fixed point. Below we investigate the properties of this optimal point of the maximal transient in more detail.

Let us consider a stimulation which is long and strong enough. If system (1), (2) is reset for a time larger than the delay $\tau$, the initial condition of system (1) at stimulus offset can be approximated by constants of the form (10) (see Sec. III). Thus, the strong reset stimulation constitutes an initial value problem for system (4) with initial values (10). We study the transient post-stimulus dynamics of the stimulusfree system (4) towards its stable phase-locked state for constant initial conditions $\varphi_{1}(t)=\varphi_{1}^{(0)}=\theta$ and $\varphi_{2}(t)=\varphi_{2}^{(0)}=(\theta$ $+\pi) / 2+\pi n, n=0,1, t \in[-\tau ; 0]$. It is easy to see that the dynamics of system (4) does not depend on the constant initial value $\varphi_{2}^{(0)}$. Hence, we fix it $\varphi_{2}^{(0)}=0$.

We explore the difference in the dynamics of system (4) for the two distinct constant initial conditions $\varphi_{1}^{(0)}=\varphi_{1}^{(u)}$ and
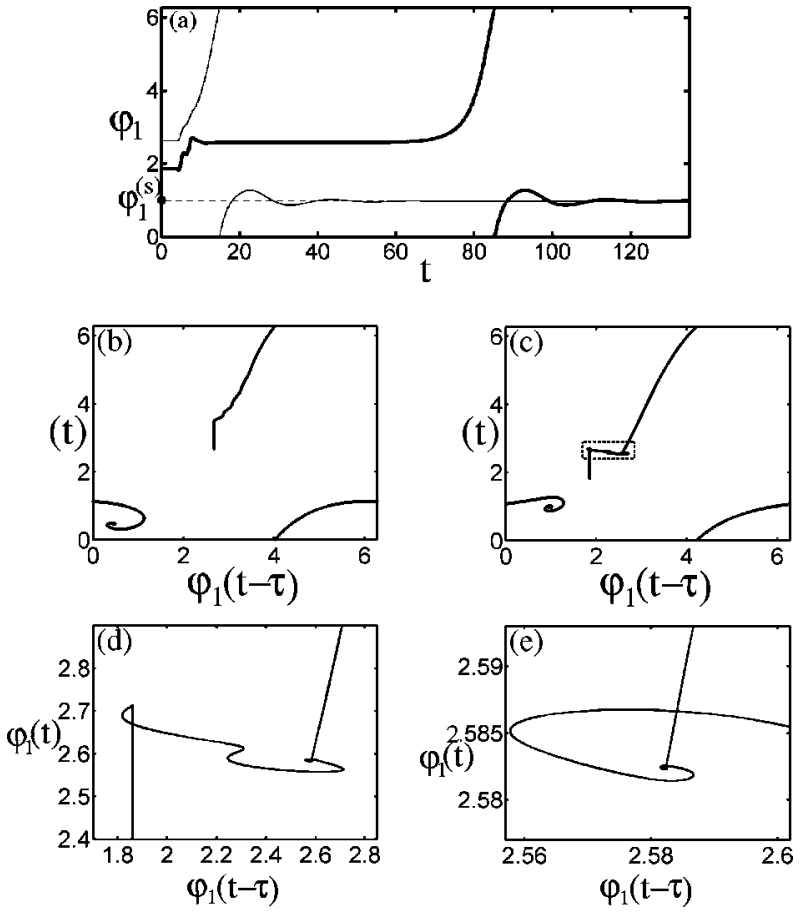

FIG. 10. (a) Transients of system (4) towards the stable fixed point $P$ occurring for two different constant initial conditions $\varphi_{1}^{(0)}$ $=2.58 \approx \varphi_{1}^{(u)}$ (thin line) and $\varphi_{1}^{(0)}=1.86 \approx \theta_{\max }$ (bold line). $\varphi_{1}^{(s)}$ and $\varphi_{1}^{(u)}$ are the $\varphi_{1}$ coordinates of the stable $P$ fixed point and the saddle fixed point $Q$, respectively. Transients from $\varphi_{1}^{(u)}$ and from $\theta_{\max }$ are shown in (b) and (c), respectively, in the $\left[\varphi_{1}(t-\tau), \varphi_{1}(t)\right]$ projection of the phase space. (d), (e) Successive enlargements of (c) around the $\varphi_{1}$ coordinate $\varphi_{1}^{(u)}$ of the unstable fixed point $Q$. Parameters: $\Delta_{1}=0.2, \Delta_{2}=3.0, \tau=4.0$, and $K=0.4$.

$\varphi_{1}^{(0)}=\theta_{\max }$. These initial states correspond to a stimulation which brings system (1), (2) into a vicinity of the unstable phase locked state $Q$ or the optimal point $\theta_{\max }$ of the maximal transient, respectively. Figure 10(a) illustrates the time course of trajectories originating from such initial conditions. The difference in the transient time for both cases is obvious: The trajectories enter an $\varepsilon$-neighborhood of the stable fixed point $P$ after transient time $T_{\text {tr }} \approx 65$ for $\varphi_{1}^{(0)}=\varphi_{1}^{(u)}$ and $T_{\text {tr }}$ $\approx 125$ for $\varphi_{1}^{(0)}=\theta_{\max }$ (with $\left.\varepsilon=0.01\right)$. Moreover, in the latter case the trajectory spends a long time in an almost stationary regime, where $\varphi_{1}(t)$ closely approaches the coordinate $\varphi_{1}^{(u)}$ of the unstable fixed point $Q$. The transients in the $\left[\varphi_{1}(t\right.$ $\left.-\tau), \varphi_{1}(t)\right]$ projection of the phase space are illustrated in Figs. 10(b)-10(d) for these two initial conditions. In the first case depicted in Fig. 10(b), where $\varphi_{1}^{(0)}=\varphi_{1}^{(u)}$, the trajectory leaves a vicinity of $Q$ and directly spirals to the stable fixed point $P$ which is a stable focus. In the second case depicted in Fig. 10(c) [with enlargements in Figs. 10(d) and 10(e)], where $\varphi_{1}^{(0)}=\theta_{\max }$, the trajectory exhibits a different itinerary: The trajectory starting at the optimal value of the initial condition $\varphi_{1}^{(0)}=\theta_{\max }$, first, very closely approaches the unstable fixed point $Q$ and stays there for a long time before it is finally attracted by $P$. Such kind of a transient takes longer than that in the first case of $\varphi_{1}^{(0)}=\varphi_{1}^{(u)}[$ Figs. 10(a) and 10(b)].

The unstable fixed point $Q=\left[\varphi_{1}(t), \varphi_{2}(t)\right]$ of system (4) has the following coordinates in the infinite-dimensional 

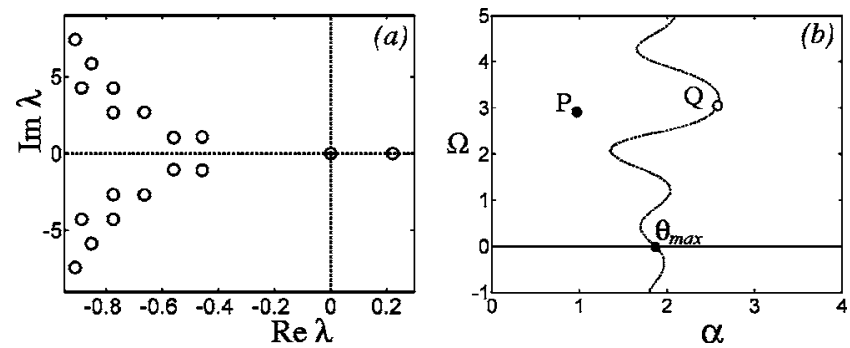

FIG. 11. (a) A few eigenvalues $\lambda_{i}$ with the largest real parts of the saddle-focus fixed point $Q$ are depicted by empty circles in the complex plane. (b) Intersection of the stable manifold $\mathcal{M}^{(s)}$ of the saddle-focus fixed point $Q$ with the class of functions (12) depicted by dashed curve in the $(\alpha, \Omega)$-parameter plane. The stable fixed point $P$ and the saddle fixed point $Q$ are shown by filled and empty circles, respectively. The stable manifold intersects the axes $\Omega=0$ at the point $\theta_{\max } \approx 1.86$. Parameters $\Delta_{1}=0.2, \Delta_{2}=3.0, \tau=4.0$, and $K$ $=0.4$.

functional phase space of Eq. (4): $\varphi_{1}(t)=\varphi_{1}^{(u)}$ and $\varphi_{2}(t)$ $=\Omega_{Q} t+$ const, where $\varphi_{1}^{(u)} \approx 2.58$ and $\Omega_{Q} \approx 3.06$ for parameter values as in Fig. 10. As mentioned above, a long and strong stimulation (2) results in an almost complete reset of system (1), (2), such that the post-stimulus transient of system (1) starts from almost constant initial conditions with $\varphi_{1}^{(0)}=\theta$ and $\varphi_{2}^{(0)}=$ const. Due to this mismatch in $\varphi_{2}$ the steady initial conditions are still far from the coordinates of the unstable fixed point $Q$ even if $\theta$ is taken equal to $\varphi_{1}^{(u)}$. Therefore, if the stimulus shifts $\varphi_{1}$ into a vicinity of $\varphi_{1}^{(u)}$, i.e., if $\theta \approx \varphi_{1}^{(u)}$, the fixed point $Q$ has a minor influence on the post-stimulus dynamics of system (1) and the transient time appears to be relatively small [Figs. $10(\mathrm{a})$ and $10(\mathrm{~b})$ ]. This is in contrast with the stimulation of coupled oscillators without delay, where the maximal transient time is attained for stimulation with $\theta=\varphi_{1}^{(u)}$ [Fig. 9(a) and Refs. [14-16,18]]. In contrast for the stimulated system with delay (1), (2), there is another optimal value of the stimulation phase shift $\theta=\theta_{\max }$, at which the transient time is essentially larger than that for the other values of $\theta$ and attains its maximum (Fig. 10). At this optimal value of the stimulation phase shift the unstable fixed point $Q$ significantly affects the post-stimulus transient dynamics, although the initial conditions of system (1) for the post-stimulus transient for $\theta=\theta_{\max }$ appear to be even more remote from $Q$ than those for $\theta=\varphi_{1}^{(u)}$.

The fixed point $Q$ of system (4) is of a saddle-focus type. Its eigenvalues are depicted in Fig. 11(a). $Q$ has one real positive eigenvalue, one zero [74], and the others are complex conjugate with negative real parts. Therefore, the fixed point $Q$ has a one-dimensional unstable manifold corresponding to the positive eigenvalue and an infinitedimensional stable manifold $\mathcal{M}^{(s)}$ corresponding to the complex eigenvalues with negative real parts. If a trajectory of system (4) comes close to $\mathcal{M}^{(s)}$, it will then follow the manifold and approach the saddle point $Q$ very closely, spending a long time there. Approaching the fixed point $Q$, the trajectory comes close to the unstable manifold of $Q$ and, thus, it will eventually be repelled from $Q$ by its unstable manifold and finally be attracted by the stable fixed point $P$. This situation is realized during the post-stimulus transient of sys- tem (1), (2) for the stimulation phase shift $\theta=\theta_{\max }$ or close to that. This can be seen in Figs. 10(c)-10(e), where the trajectory of Eq. (4) spirals to $Q$ following $\mathcal{M}^{(s)}$, thereby exploring a characteristic focus shape of the stable manifold of $Q$, and only thereafter that being attracted by $P$.

The itinerary of the trajectory shown in Figs. 10(c)-10(e) reflects the property of the saddle fixed point $Q$ that its stable manifold $\mathcal{M}^{(s)}$ has an intersection with a class of constant initial conditions of system (4). To illustrate this fact, consider the following class of linear functions

$$
\psi_{1}(t)=\Omega t-\alpha / 2, \quad \psi_{2}(t)=\Omega t+\alpha / 2 .
$$

Taking functions (12) as initial conditions for system (1) we find regions in the $(\alpha, \Omega)$-parameter space, where the trajectories demonstrate different transients on their ways towards the stable fixed point $P$ born in a saddle-node bifurcation together with $Q$. The transients differ with respect to whether or not the variable $\varphi_{1}$ rotates once through a cycle of $2 \pi$ before being attracted by P. In other words, we find a boundary between basins of attraction of $P$ and its copy shifted by $2 \pi$. This boundary is depicted in Fig. 11(b) by a dotted curve in the $(\alpha, \Omega)$-parameter space. It goes through the saddle fixed point $Q$ and is the intersection of the stable manifold $\mathcal{M}^{(s)}$ of $Q$ with the class of functions (12). The manifold serves as separator between the two different kinds of poststimulus transients of system (1). The stable manifold also intersects with the axis $\Omega=0$, i.e., it intersects with the class of constant initial conditions of system (1) at the point $\alpha$ $=\theta_{\max } \approx 1.86$ which is the phase shift of the maximal poststimulus transient considered above (Fig. 10). This demonstrates the central role of the stable manifold $\mathcal{M}^{(s)}$ of the saddle point $Q$ in the post-stimulus dynamics of the stimulated system (1), (2).

The existence of the intersection point $\theta_{\max }$ of the line $\{\Omega=0\}$ with the stable manifold of $Q[$ Fig. 11(b) $]$ means that for a long and strong stimulation of system (1), (2) there exists an optimal value of the stimulation phase shift $\theta_{\max }$ such that for $\theta \approx \theta_{\max }$ a very long post-stimulus transient occurs. Theoretically, $T_{\operatorname{tr}} \rightarrow \infty$ as $\theta \rightarrow \theta_{\max }$, and when the post-stimulus initial conditions directly fit the stable manifold $\mathcal{M}^{(s)}$ of the fixed point $Q$. The trajectory will then be directly attracted by $Q$ and will never relax to the stable fixed point $P$. Put otherwise, the trajectory gets trapped by the stable manifold $\mathcal{M}^{(s)}$. However, this limiting case is difficult to be realized in practice, because of the inevitable noise and the mismatches in the stimulus-induced reset. Nevertheless, even with noise, the transient time at $\theta=\theta_{\max }$ or close to $\theta_{\max }$ is significantly larger than that for the other values of $\theta$. This is illustrated in Fig. 12, where a few trajectories of system (1), (2) stimulated with noise and stimulation phase shifts $\theta=\varphi_{1}^{(u)}\left[\right.$ Fig. 12(a)] or $\theta=\theta_{\max }$ [Fig. 12(b)] are plotted. Comparing the results of the calculations shown in Fig. 12 with those in Fig. 10, one finds that the trajectories still follow the corresponding itineraries as in the case of ideal constant initial conditions, which provides evidence for longer transients for the optimal value of the stimulation phase shift $\theta=\theta_{\max }$.

The optimal stimulation phase shift $\theta_{\max }$ has another important property. In addition to causing of the maximal transient time, $\theta_{\max }$ is also connected with maximal clustering of 

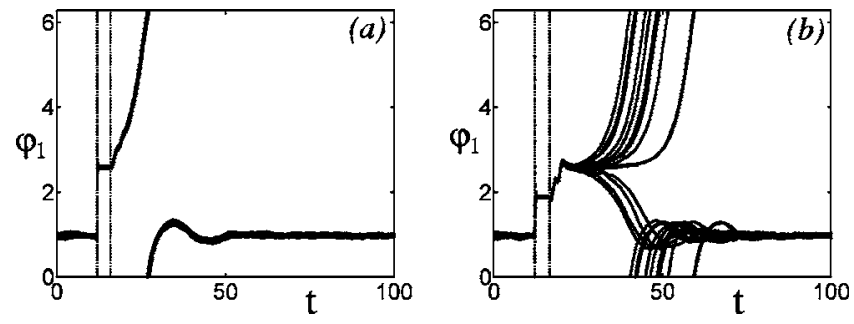

FIG. 12. Post-stimulus transients of system (1), (2) stimulated with (a) $\theta=2.583 \approx \varphi_{1}^{u}$ and (b) $\theta=1.87 \approx \theta_{\max }$ and with Gaussian white noise with $D=0.001$. Parameters $I=15.0, T_{\mathrm{st}}=4.5, \Delta_{1}=0.2$, $\Delta_{2}=3.0, \tau=4.0$, and $K=0.4$. The number of stimuli $N=15$ in both plots.

the post-stimulus responses of system (1), (2) in the $\varphi_{1}$ variable (phases $\psi_{1}$ and $\psi_{2}$, respectively). The clustering of the post-stimulus responses can be seen in Figs. 5(a), 5(c), 5(e), and 12(b). The clustering measure $H$, i.e., the relative number of trajectories within the smallest cluster in variable $\varphi_{1}$ for the post-stimulus transient is plotted in Fig. 13 versus stimulation phase shift $\theta$. Maximal clustering is attained at $\theta=1.87 \approx \theta_{\max }$ which is very close to the theoretically obtained point of the maximal post-stimulus transient (Figs. 10 and 11). This phenomenon is connected with the properties of the stable manifold $\mathcal{M}^{(s)}$ of the saddle-focus fixed point $Q$. The manifold serves as separator between two different kinds of the post-stimulus transients of system (4). In this connection, the optimal value $\theta_{\max }$ can also be referred to as a separator of the post-stimulus trajectories of system (4). Indeed, as mentioned above, for the stimulation phase shift $\theta \approx \theta_{\max }$ the trajectories starting very close to $\mathcal{M}^{(s)}$ approach $\mathcal{M}^{(s)}$, follow it towards $Q$ and stay there for a long time. After a long attraction phase they will be repelled from $Q$ and attracted by the stable fixed point $P$. On the other hand, if $\theta \neq \theta_{\max }$ then the trajectory of system (4) will be directly attracted by $P$ or by a copy of $P$ shifted by $2 \pi$ without or with an additional rotation on the torus, respectively. For example, for parameter values as in Fig. 13, stimulation with

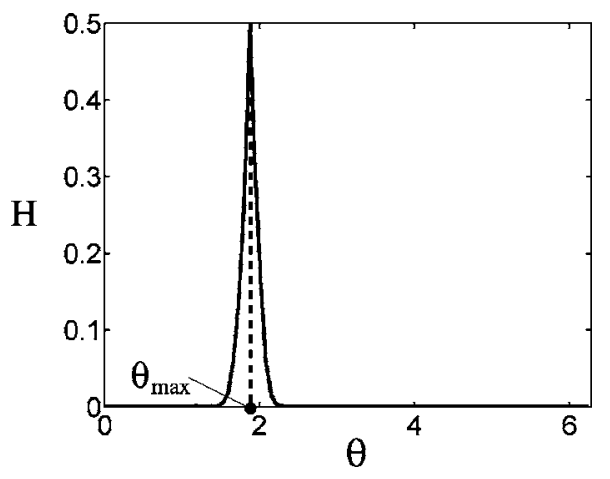

FIG. 13. Post-stimulus clustering measure $H$, i.e., the relative number of trajectories in the smallest cluster of the post-stimulus responses in variable $\varphi_{1}$ versus stimulation phase shift $\theta$. System (1), (2) is simulated with Gaussian white noise of amplitude $D$ $=0.002$. The width of the peak of the measure depends on the amplitude of the noise and grows with an increase of the noise amplitude. Number of stimuli $N=100$, stimulus length $T_{\mathrm{st}}=5$, and other parameters: $\Delta_{1}=0.2, \Delta_{2}=3.0, \tau=4.0$, and $K=0.4$. $\theta<\theta_{\text {max }}$ leads to a simple post-stimulus transient, where trajectories are directly attracted by $P$. In contrast, stimulation with $\theta>\theta_{\max }$ results in that the post-stimulus trajectories perform one rotation in $\varphi_{1}$ on the torus and will be attracted by a copy of $P$ shifted by $2 \pi$. In this way, when stimulating with $\theta \approx \theta_{\max }$ in the presence of noise, there will be trajectories relaxing to $P$ after stimulus offset according to the first scenario $\left(\theta<\theta_{\max }\right)$, and there will be trajectories relaxing to $P$ according to the second scenario $\left(\theta>\theta_{\max }\right)$, see Fig. 12(b). The relative number of trajectories in each of such clusters can be relatively close to each other (Fig. 13). This explains the formation of the post-stimulus clustered responses of variable $\varphi_{1}$ of the stimulated system with delay (1), (2), see Fig. 5.

Simultaneously with the formation of the post-stimulus response clusters of the variable $\varphi_{1}$, clusters also emerge of the phase variables $\psi_{1}$ and $\psi_{2}$, see Fig. 5. Variable $\varphi_{2}$, in turn, gets clustered during stimulation and keeps being clustered after stimulus offset. In the time course after stimulus offset, the response clusters in variable $\varphi_{1}$ are suppressed when the trajectories are attracted by the stable phase-locked state, see also Fig. 12(b). In contrast, the clusters in the phase variables $\psi_{1}$ and $\psi_{2}$ are preserved for the whole post-stimulus transient period. The clustering of the variable $\varphi_{1}$ means that some trajectories are approaching the value $\varphi_{1}^{(s)}$, and the others are approaching $\varphi_{1}^{(s)} \pm 2 \pi$, where $\varphi_{1}^{(s)}$ is the $\varphi_{1}$ coordinate of the stable fixed point $P$. Taken $\bmod 2 \pi$ these clusters are not seen in Figs. 5(e) and 5(b). Simultaneously, across trials the variable $\varphi_{2}$ attains two different values from Eq. (10) at stimulus offset. Assuming that the clustering in $\varphi_{2}$ is preserved, with a simple calculation one arrives at the conclusion that the clustering in variable $\varphi_{1}$ determines the clustering of the phase variables $\psi_{1}$ and $\psi_{2}$ : Two trajectories from different clusters in $\varphi_{1}$ are also from different clusters in the phase variables $\psi_{1,2}$ and vice versa. Moreover, the "distance" between the clusters in the phase variables is $\pi$ and the clusters are clearly observed in the cross-trial diagram in Figs. 5(a) and 5(c). Therefore, the optimal stimulation phase shift $\theta_{\max }$ serves both for maximal post-stimulus transient time and for maximal post-stimulus response clustering of system (1).

\section{B. Stimulation of periodically modulated synchronized states}

A phase-locked state of system (1) is stable only for a finite interval of parameter $K$ values. Exemplary phaselocked states (also called fixed points) $P$ and $Q$ [Fig. 2(a)] have been considered above. When the coupling parameter $K$ increases, the stable fixed point $P$ loses its stability via a supercritical Hopf bifurcation at which a stable limit cycle $\gamma$ is born [Figs. 2(a) and 2(c)]. The limit cycle $\gamma$ is stable within a range of the coupling strength. In this regime, called periodically modulated phase synchronization, the phase difference $\varphi_{1}$, being attracted by $\gamma$, is periodically oscillating and is bounded between its maximal $\varphi_{1}^{\max }$ and minimal $\varphi_{1}^{\min }$ values [Figs. 2(a), 2(c)]. In this section we study the influence of stimulation (2) on system (1) in the dynamical regime of periodically modulated phase synchronization given by the limit cycle $\gamma$. 

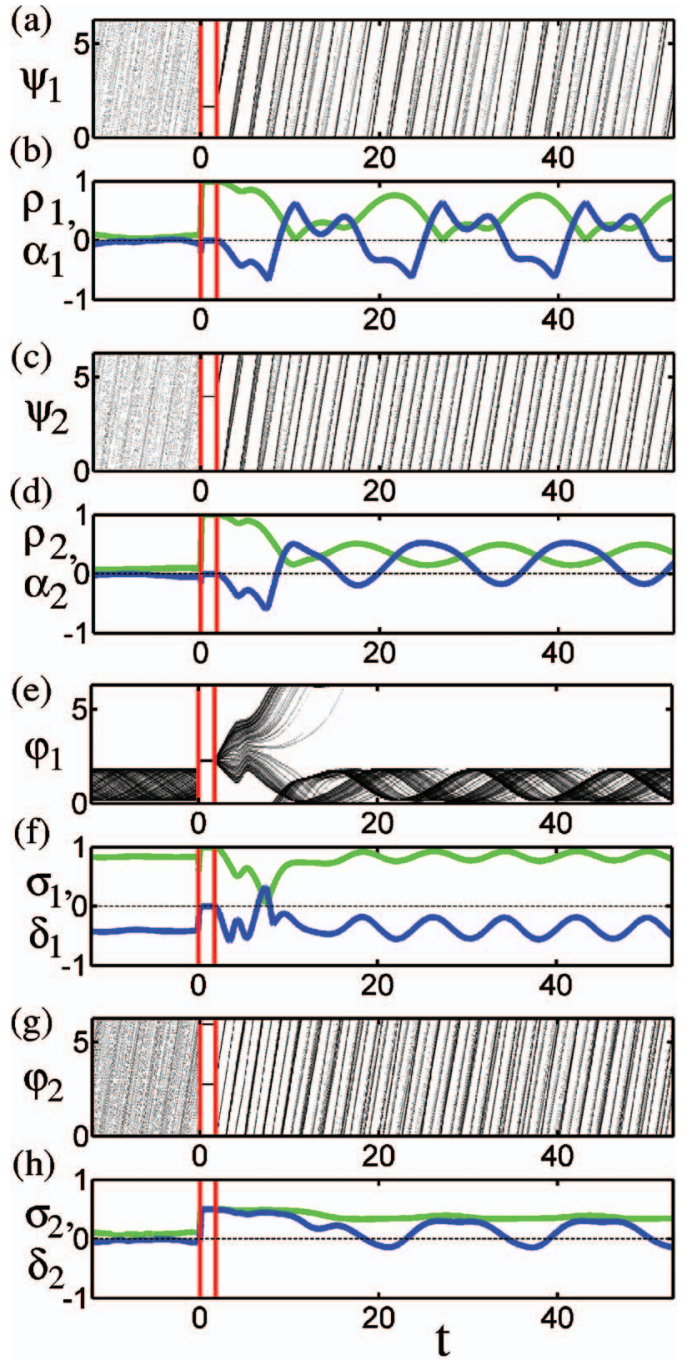

FIG. 14. (Color) Stimulus-locked responses of system (1) stimulated in a periodically modulated synchronized regime with signal (2). Cross-trial distributions of the phases $\psi_{1}, \psi_{2}$, the phase difference $\varphi_{1}$, and the mean phase $\varphi_{2}$ are shown in (a), (c), (e), and (g), respectively. The density of trajectories is encoded with the intensity of gray. Onsets and offsets of stimuli are indicated by vertical red lines. The features of stimulus-locked dynamics as revealed by the stochastic phase response analysis (see the Appendix and Refs. [14-16,18]): The resetting indices $\rho_{1}, \rho_{2}$ and the clustering indices $\alpha_{1}, \alpha_{2}$ of the phases $\psi_{1}$ and $\psi_{2}$ are shown in (b) and (d); the resetting indices $\sigma_{1}$ and $\sigma_{2}$ and the clustering indices $\delta_{1}$ and $\delta_{2}$ of the phase difference $\varphi_{1}$ and the mean phase $\varphi_{2}$ are shown in (f) and (h). The resetting/synchronization indices (green curves) and the clustering indices (blue curves) detect unimodal and antiphase bimodal cross-trial distributions, respectively. The number of stimuli $N$ $=300$. Parameters $\Delta_{1}=0.2, \Delta_{2}=3.0, \tau=4.0, K=0.7$, and $\theta=2.27$.

An example of the cross-trial distributions and the corresponding stimulus locking indices (see the Appendix) typical for stimulation in this regime is shown in Fig. 14. Due to the randomized stimulus administration, in the prestimulus time interval the phase difference $\varphi_{1}$ fills the whole segment $\left[\varphi_{1}^{\min }, \varphi_{1}^{\max }\right]$. A strong and long enough stimulus (2) results in the phase reset as described in Sec. IV. The coordinates of the reset states (10) are determined by the stimulation phase shift $\theta$ and are independent of the underlying dynamics of system (1). For the considered case of a stimulated limit cycle, the in- and post-stimulus transients are more complicated than those for the case of stimulated phase-locked states considered above. For the in-stimulus transient, for instance, the analysis similar to that from Sec. IV can be applied. The difference here is that the initial conditions of system (4) summarized over all stimulation trials at the stimulus onsets fill the whole stripe $\left[\varphi_{1}^{\min }, \varphi_{1}^{\max }\right] \times[0,2 \pi)$ in the phase space in Fig. 6. This stripe intersects the basins of attraction of the stable reset states $A$ and $B$ of system (8) and, hence, a more complicated clustered in-stimulus transient in system (1), (2) can be observed.

When the stimulation is off, the trajectories of system (1) relax from the reset state to the stable limit cycle $\gamma$. As discussed above, the stable manifold $\mathcal{M}^{(s)}$ of the saddle-focus fixed point $Q$ of system (4) serves as separator of two kinds of post-stimulus dynamics of system (1), (2) when it is stimulated in a phase-locked state, see Sec. V A. The same holds for the stimulated regime of periodically modulated phase synchronization, since the saddle fixed point $Q$ coexists with the limit cycle $\gamma$. The difference here is that the trajectories of Eq. (4) will be attracted after stimulus offset by the limit cycle. This is illustrated by Fig. 14, where system (1), (2) is stimulated with an optimal stimulation phase shift $\theta \approx \theta_{\max }$. One can see that there are two different groups of trajectories splitting from each other when the stimulus is off. The $\varphi_{1}$ coordinate of one of them is decaying, so that the trajectories are directly attracted by $\gamma$. The other group of trajectories exceeds the value $\varphi_{1}=2 \pi$ and is attracted by a copy of $\gamma$ shifted by $2 \pi$. This mechanism, which is similar to the case of the stimulated phase-locked states, generates two stereotypical post-stimulus system responses.

With a more detailed consideration of the post-stimulus dynamics of system (4) one observes another important phenomenon. For the post-stimulus responses, in addition to splitting trajectories into two post-stimulus clusters, there is also an additional spreading of them within each of the clusters of variable $\varphi_{1}$ [Fig. 14(e)]. Such kind of spreading can also be observed for the stimulated phase locked-state in a short time interval after stimulus offset, see Figs. 5(e) and 12(b). However, for the stimulated phase-locked states, in the course of the post-stimulus transient these broad clusters of variable $\varphi_{1}$ are suppressed, when the trajectories are attracted by a stable fixed point. For the case of stimulated periodically modulated synchronized state, the clusters of variable $\varphi_{1}$ are preserved for the whole post-stimulus transient period. Moreover, the spreading of trajectories within the clusters is preserved as well [Fig. 14(e)]. This phenomenon is strongly related to the properties of dynamics on a limit cycle.

The initial conditions of system (4) at stimulus offset slightly differ from each other because of noise, mismatches in reset states, and time delay (if the phases are reset for a time shorter than delay). Therefore, each trajectory spends a different amount of time during post-stimulus transient before it is attracted by the limit cycle $\gamma$. This results in that the trajectories approaching $\gamma$ attain different phase shifts along the limit cycle with respect to each other. Since there is no contraction or expansion along $\gamma$, the trajectories will preserve their phase shifts after a complete rotation around $\gamma$, 
which can clearly be seen in Fig. 14(e). This explains the mechanism of creation of the multicluster stimulus response of system (1), (2) stimulated at the periodically modulated synchronized state.

The phenomena above are clearly reflected by resetting and clustering indices depicted in Fig. 14. Indeed, the resetting and clustering indices of the phases $\psi_{1}$ and $\psi_{2}$ as well as of the phase difference $\varphi_{1}$ (see the Appendix) exhibit periodic oscillations preserved for the whole post-stimulus transient period. Such periodic oscillations of the stimulus locking indices are caused by periodic oscillations of clusters of trajectories of system (1) on the limit cycle $\gamma$. Within a period of rotation on $\gamma$, the projections of the response clusters on the corresponding axes demonstrate a subsequent gathering and separation of the clusters, leading to an antiphase oscillation of the resetting and clustering indices, respectively. Such a dynamics of stimulus locking indices of the phases $\psi_{1}$ and $\psi_{2}$ can be seen in Figs. 14(b) and 14(d). The state of clusters and values of stimulus locking indices are repeated after each period of the oscillations of trajectories on $\gamma$. In general, this results in a periodic behavior of the resetting and clustering indices with the same period as that of the limit cycle $\gamma$ [Figs. 14(b) and 14(d)].

\section{POST-STIMULUS DYNAMICS IN MULTISTABLE REGIMES}

Multistability is a common phenomenon in complex systems, in particularly, in systems with delay $[23,28,30,31]$. In this section we consider the impact of stimulation (2) on system (1) stimulated in dynamical regimes, where more than one stable state exists.

\section{A. Bistability of phase-locked states}

The first considered example is the case of two coexisting stable phase-locked states, denoted by $P$ and $P^{\prime}$, shown in Fig. 3. In both states the phases are in-phase locked with $\varphi_{1}^{*}=0$. However, $P$ and $P^{\prime}$ have different frequencies: $\Omega_{P}$ $\approx 5.12$ and $\Omega_{P^{\prime}} \approx 3.96$, respectively. Depending on initial conditions, a trajectory of system (1) will be attracted by $P$ or by $P^{\prime}$. We calculate the basins of attraction of $P$ and $P^{\prime}$ for the class of initial conditions of the form (12) (see also Ref. [28]). The parameters to be varied here are the phase shift $\alpha$ and the frequency $\Omega$. In Fig. 15, the basins of attraction of the fixed points $P$ (white region) and $P^{\prime}$ (gray regions) are shown in the $(\alpha, \Omega)$-parameter plane. The stable fixed points $P$ and $P^{\prime}$ are depicted by black filled circles and the corresponding saddle fixed points $Q$ and $Q^{\prime}$, which are born with $P$ and $P^{\prime}$ in saddle-node bifurcations, respectively, are depicted by empty circles.

As mentioned above, after long and strong enough stimulation (2), a post-stimulus transient in system (1) starts from almost constant initial condition (see Sec. IV). Therefore, the post-stimulus initial conditions of Eq. (1) are located in Fig. 15 within a small stripe with $\Omega \approx 0$ and with the phase shift $\alpha=\theta$. By choosing an appropriate stimulation phase shift $\theta$ one can put the initial conditions of system (1) for the poststimulus transient into one or the other basin of attraction. In
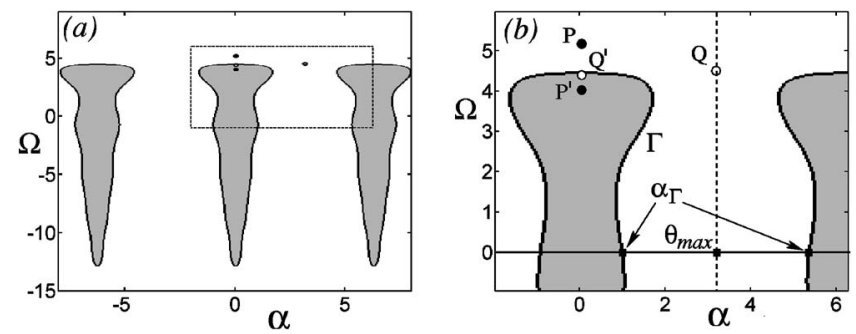

FIG. 15. Basins of attraction of two stable phase-locked states $P$ (white region) and $P^{\prime}$ (gray regions) of system (1) calculated for initial conditions of the form (12) in the $(\alpha, \Omega)$-parameter plain. (b) Enlargement from (a). The stable fixed points $P$ and $P^{\prime}$ are indicated by filled circles and the saddle fixed points $Q$ and $Q^{\prime}$ are indicated by empty circles. $\Gamma$ is the basin boundary of $P^{\prime}$ and $\alpha_{\Gamma}$ $=\Gamma \cap\{\Omega=0\}, \alpha_{\Gamma} \approx 0.95$, and $\alpha_{\Gamma} \approx 2 \pi-0.95$. The vertical dashed line shows the intersection of the stable manifold of $\mathrm{Q}$ with the class of functions (12), where $\theta_{\max }=\pi$. Parameters: $\Delta_{1}=0.0, \Delta_{2}$ $=4.5, \tau=1.4$, and $K=1.6$.

this way the stimulation can redirect trajectories from one to the other stable state, e.g., from the phase-locked state $P$ to $P^{\prime}$ and vice versa. This is illustrated in Fig. 16, where the stimulation is performed with two different phase shifts $\theta$ $=0.9$ and $\theta=1.0$ resulting in different synchronized dynamics after stimulus offset, respectively. Note, the stimulus-induced switching between the states $P$ and $P^{\prime}$ does not manifest itself in a change of the synchronization properties from the preswitching to the postswitching dynamics of system (1): In both states the oscillators are in-phase synchronized. However, after the switching the mean frequency of the synchronized state is significantly different.

The basin boundary $\Gamma$ (boundary between gray and white regions in Fig. 15) plays an important role in the structure of the basin of attraction. Taking initial conditions close to $\Gamma$, one observes that the trajectory of system (4) very closely approaches the unstable fixed point $Q^{\prime}$. For such initial conditions the transient dynamics is very similar to that discussed in Sec. V A, see Fig. 10. As mentioned above, the fixed point $Q^{\prime}$ is born in a saddle-node bifurcation with $P^{\prime}$ and is of a saddle-focus type. $Q^{\prime}$ has one real and positive eigenvalue, one zero, and the others are complex conjugate
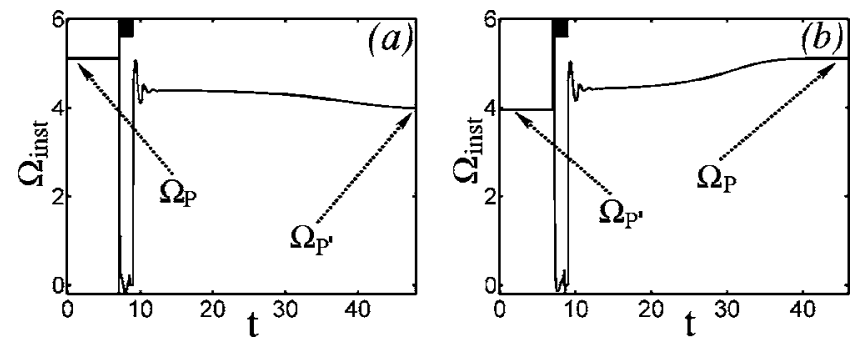

FIG. 16. Stimulus-induced switching between the two stable in-phase-locked states $P$ and $P^{\prime}$ from Fig. 3: (a) Stimulation phase shift $\theta=0.9$ induces a transition from $P$ with frequency $\Omega_{P} \approx 5.12$ to $P^{\prime}$ with frequency $\Omega_{P^{\prime}} \approx 3.96$, and (b) stimulation phase shift $\theta$ $=1.0$ induces the inverse transition from $P^{\prime}$ to $P$. The vertical axis stands for the an instantaneous mean frequency $\Omega_{\text {inst }}=\dot{\varphi}_{2}$. Stimulation epochs are indicated by black bars at the top of the graphs. Parameters: $I=15, T_{\mathrm{st}}=2.0, \Delta_{1}=0.0, \Delta_{2}=4.5, \tau=1.4$, and $K=1.6$. 


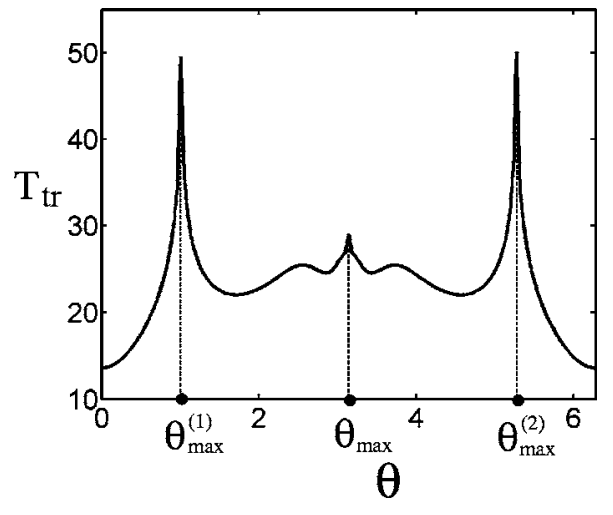

FIG. 17. Mean post-stimulus transient time $T_{\text {tr }}$ versus the stimulation phase shift $\theta$ calculated for the stimulated system (1), (2). Three local maxima are observed for $\theta=\theta_{\max }^{(1)}, \theta_{\max }$, and $\theta_{\max }^{(2)}$, corresponding to the three points which closely approach the stable manifolds of saddle fixed points $Q\left(\theta_{\max }\right)$ and $Q^{\prime}\left(\theta_{\max }^{(1)}\right.$ and $\left.\theta_{\max }^{(2)}\right)$ (see also Fig. 15). The number of stimuli $N=100$. Parameters $\Delta_{1}$ $=0.0, \Delta_{2}=4.5, \tau=1.4, K=1.6, I=25$, and $T_{\mathrm{st}}=2.5$.

with negative real parts. The point $Q^{\prime}$ is placed on $\Gamma$ and thus, its stable manifold $M^{(s)}\left(Q^{\prime}\right)$ contributes to the basin boundary and serves as separator between basins of attraction of two different stable phase-locked states $P$ and $P^{\prime}$. In this way, the basin boundary $\Gamma$ shown in Fig. 15 represents an intersection of the stable manifold $M^{(s)}\left(Q^{\prime}\right)$ with a class of functions (12). Therefore, analogously to the case considered in Sec. V A, one may expect that a very long poststimulus transient in system (1), (2) may occur also for the case illustrated in Fig. 15. Indeed, from Fig. 15 it follows that the basin boundary $\Gamma$ intersects the line $\Omega=0$ in points denoted by $\alpha_{\Gamma}$. Since the post-stimulus initial conditions very closely approach constant functions, they can be adjusted to very closely approach the point $\alpha_{\Gamma} \in \Gamma$ (Fig. 15). This can be achieved by strong and long enough stimulation with phase shift $\theta \approx \alpha_{\Gamma}$. Then, during the post-stimulus transient, the trajectories of system (1) will follow the stable manifold $M^{(s)}$ of $Q^{\prime}$ approaching the saddle point $Q^{\prime}$ and spend a long time there before being attracted by one of the stable fixed points Por $P^{\prime}$.

For the parameter values as in Fig. 15, there exists another saddle-focus fixed point $Q$ which is born simultaneously with $P$. The stable manifold $M^{(s)}(Q)$ of the fixed point $Q$ also has an intersection with the class of initial functions (12) and with the line $\Omega=0$ [dashed line in Fig. 15(b)], which has been discussed in Sec. V A. Therefore, there is another optimal value of the phase shift $\alpha=\theta_{\max }$ such that stimulation with phase shift $\theta \approx \theta_{\max }$ brings the post-stimulus initial conditions of system (1) close to $M^{(s)}(Q)$. The corresponding post-stimulus trajectories then approach the saddle fixed point $Q$, which results in a long post-stimulus transient towards the stable phase-locked state $P$, similar to that illustrated in Fig. 10.

The length of the mean post-stimulus transient time $T_{t r}$ calculated for the stimulated system (1), (2) for parameter values from Fig. 15 is depicted in Fig. 17 versus stimulation phase shift $\theta$. One can see that there are three optimal values of $\theta=\theta_{\max }^{(1)}, \theta_{\max }^{(2)}$, and $\theta_{\max }$, where the transient time $T_{\text {tr }}$ attains

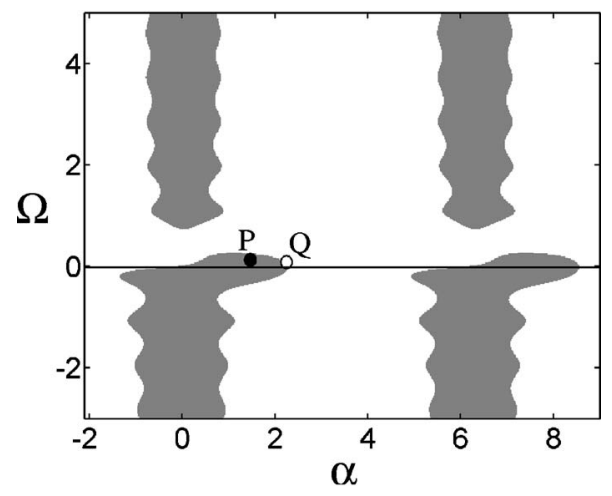

FIG. 18. Basins of attraction of the stable phase-locked state $P$ (gray regions) and the stable desynchronous limit cycle $\mu$ (white regions) of system (1) [Fig. 4(b)] calculated for initial conditions of the form (12) in the $(\alpha, \Omega)$-parameter plain. The black filled circle indicates the stable fixed point $P$ and the empty circle indicates the saddle fixed point $Q$. Parameters $\Delta_{1}=0.2, \Delta_{2}=0.1, \tau=7.2$, and $K$ $=0.28$.

local maxima. These are the points, where the post-stimulus initial conditions of system (1) come close to the stable manifolds $M^{(s)}\left(Q^{\prime}\right)$ and $M^{(s)}(Q)$ of the fixed points $Q^{\prime}$ and $Q$, respectively. More precisely, the optimal phase shifts $\theta_{\max }^{(1,2)}$ correspond to the phase shifts $\alpha_{\Gamma}$ (Fig. 15), which are placed on the basin boundary $\Gamma$ and, thus, belong to the stable manifold $\mathcal{M}^{(s)}\left(Q^{\prime}\right)$. Stimulation with such phase shifts directs the trajectories during the post-stimulus transient to the saddle fixed point $Q^{\prime}$. The optimal phase shift $\theta_{\max }$ corresponds to the stable manifold $M^{(s)}(Q)$, and stimulation with such a phase shift directs the trajectories to the saddle fixed point $Q$, see also Sec. V A.

\section{B. Bistability of synchronous and desynchronous dynamics}

Multistability in system (1) occurs not only between synchronized states, but also between synchronized and desynchronized states, as shown in Sec. III (see Fig. 4). An example of a stable phase-locked state $P$ which coexists with the stable desynchronized limit cycle $\mu$ is shown in Fig. 4(b). In this section we consider the impact of stimulation (2) on the dynamics of system (1) when it exhibits a multistable regime with the stable point $P$ and a cycle $\mu$. We consider parameter values indicated by point $A$ in Fig. 4(a).

With the use of the class of linear functions (12), in Fig. 18 the basins of attraction of the stable phase-locked state $P$ (gray regions) and the desynchronous limit cycle (white region) are shown in the $(\alpha, \Omega)$-parameter plane. Stimulation resets the variables of system (1) to the almost constant functions (Sec. IV). Therefore, as before, the initial conditions of system (1) for the post-stimulus transient are located close to the line $\Omega=0$ with $\alpha=\theta$ in the $(\alpha, \Omega)$-parameter plane in Fig. 18. By varying the stimulation phase shift $\theta \in[0 ; 2 \pi)$, the initial conditions can be placed at any point on the horizontal axis $\Omega=0$. As one can see, this line intersects both basins of attraction in Fig. 18 and, thus, stimulation (2) with appropriate phase shift $\theta$ can redirect the dynamics of the system from one stable state to the other. 

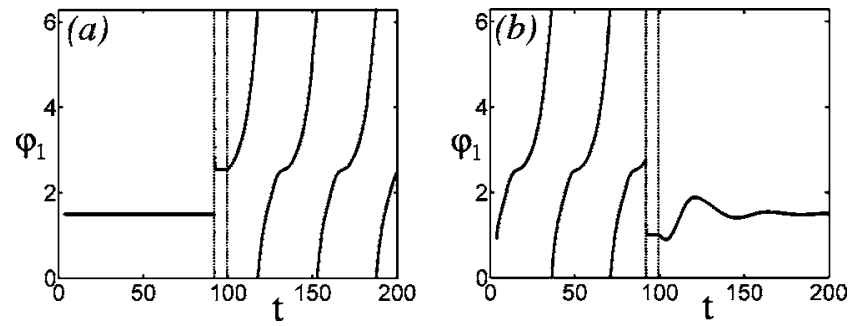

FIG. 19. Stimulus-induced switching between the stable phaselocked state $P$ and the stable desynchronous limit cycle $\mu$ [Fig. 4(b)]: (a) Stimulation phase shift $\theta=2.3$ induces a transition from $P$ to $\mu$, and (b) stimulation phase shift $\theta=2.2$ induces an inverse transition from $\mu$ to $P$. Stimulation onsets and offsets are indicated by vertical dashed lines. Parameters: $I=15.0, T_{\mathrm{st}}=8.0, \Delta_{1}=0.2, \Delta_{2}$ $=0.1, \tau=7.2$, and $K=0.28$.

The switching between synchronized and desynchronized states caused by stimulation is illustrated in Fig. 19. Starting in a synchronized phase-locked regime, stimulation can effectively desynchronize system (1) [Fig. 19(a)]. On the other hand, starting from the desynchronized dynamics, the stimulation can also induce phase-locked synchronization [Fig. 19(b)]. Applying multiple stimuli one can end up with two different situations: (i) All trajectories before and after stimulation will exhibit the same synchronized or desynchronized dynamics, respectively, or (ii) there will be a mixture of trajectories, attracted by both stable synchronized and desynchronized states.

\section{DISCUSSION}

We studied stimulus-locked responses of two phase oscillators coupled with delayed self-feedback. For this, we first investigated the spontaneous (stimulation-free) dynamics of our model equation (1). Our analysis shows that it makes a big difference whether two phase oscillators are coupled with a delayed self-feedback [Eq. (1)] or with a transmission delay [28]. In phase oscillators coupled with a transmission delay a variety of phase locked states occurs, depending on model parameters [28]. In contrast, the delayed self-feedback from Eq. (1) leads to several qualitatively different dynamical regimes, ranging from desynchronized motion, phase locking, periodically modulated synchronization to chaotic phase synchronization, emerging in dependence on model parameters.

The spontaneous dynamics of the two coupled PLLs has a remarkable feature. In phase oscillators coupled either without delay [51-53] or with a transmission delay [28,31] strong coupling guarantees synchronization: A transition to synchronization can be achieved by means of a sufficiently strong increase of the coupling strength. What we found in our model, in two phase oscillators coupled with a delayed self-feedback [Eq. (1)], is the direct opposite. For sufficiently strong coupling strength the oscillators become desynchronized. This is particularly intriguing since this model equation stands for two coupled PLLs (Fig. 1), which are two generic phase-locking devices, that are supposed to mutually adapt their phases. In fact, this result illustrates that the ac- tion of a PLL with internal delay decisively depends on the structures it interacts with (see also Refs. [39,40]).

The essential ingredient of our model is a time-delayed self-feedback. This mechanism is relevant in the nervous system, as large-scale feedback loops constitute an important class of neuronal circuits, which are involved in closed-loop computations and optimized signal processing, e.g., at the level of thalamocortical and corticocortical sensorimotor loops [44,46-48]. Also, to compensate for errors in movement execution, the cerebellum receives a self-feedback signal with a delay from the muscles, which conveys information about actually performed movements [54]. Closed loop mechanisms and delayed feedback are not only relevant for neural networks $[19,22]$, biology $[21,23]$, and neuroscience $[20,40,55]$, but also for laser physics [56,57] and other fields of physics [31,32,34,35,39,58-61].

Using a stochastic phase response analysis [14-16,18] and bifurcation theory we studied the dynamical mechanisms underlying the stimulus-locked responses. In particular, this enabled us to explain the formation of in- and post-stimulus response clustering of the phase variables in the system's phase-locked regime. While a stimulus of sufficient strength determines the in-stimulus response clustering completely (Sec. IV), the post-stimulus dynamics crucially depends on the model's dynamical regime (Secs. V and VI).

Previous studies devoted to stimulus-locked responses of two phase oscillators coupled without delay where carried out with different stimulation schemes and coupling schemes [14-16,18]: Apart from first order stimulation terms, such as $S_{j}\left(\psi_{j}\right)=X(t) I \cos \left(\psi_{j}-\theta_{j}\right)$ [see Eq. (2)], also stimulation terms of higher order, such as $S_{j}\left(\psi_{j}\right)=X(t) I \cos \left(2 \psi_{j}\right)$, have been studied [17]. Not only lowest-order coupling terms, such as $\sin \left(\psi_{2}-\psi_{1}\right)$ [see Eq. (1)], but also bistable, higher-order coupling terms, such as $\sin \left(\psi_{2}-\psi_{1}+\theta\right)-\sin \left[2\left(\psi_{2}-\psi_{1}+\theta\right)\right]$ $-\sin \left[4\left(\psi_{2}-\psi_{1}+\theta\right)\right]$, have been taken into account [18]. In all of these studies pulsatile stimuli (with appropriate parameters) caused a phase reset or a response clustering or a phase reset followed by a response clustering [14-16,18].

The two phase oscillators coupled with delayed selffeedback [Eq. (1)] may show similar, but also novel stimulus-locked responses. The type of the response depends on the dynamical regime, which, in turn, depends on the choice of model parameters. In the phase-locked regime stimuli with appropriate parameters elicit a phase reset followed by an antiphase response clustering, comparable to what has been found for two phase oscillators coupled without delay $[14-16,18]$. In contrast, in the regime of periodically modulated phase synchronization the two phase oscillators coupled with delayed self-feedback [Eq. (1)] behave completely different: We observe a stimulus-locked oscillation between phase reset and response clustering (with two anti-phase response clusters), which is combined with an oscillatory modulation of the stimulus-locked synchronization (Fig. 14).

Reverberating phase resets of this kind might be relevant in the context of neural responses evoked by visual stimuli. Already simple visual stimuli (like black and white checkerboard patterns) typically elicit not just a single response, but a sequence of responses, as revealed by CTA [62-65]. The 
mechanism underlying the generation of reverberating cortical activations of this kind is still a matter of debate, where possible components might be feedback mechanisms in cortico-cortical and cortico-subcortical loops (see, e.g., Ref. [65]).

Multistability is a phenomenon which is typically observed in systems with time delay $[23,28,30,31,34,35]$. Also in our model, given by Eq. (1), we observe not only unistable dynamical regimes, but also multistability. We demonstrate how appropriate stimuli may shift the systems dynamics either from one stable synchronized state to another synchronized state with a different frequency or from a synchronized to a desynchronized state. Massive long-term changes of the dynamics caused by a single-shot stimulus may be relevant in the context of novel deep brain stimulation techniques which aim at specifically desynchronizing pathological neural synchrony in particular target areas in neurological disorders such as Parkinson's disease or epilepsy $[66,67]$.

Another interesting finding in this context is the dependence of the maximal transient time (Sec. V A) on the phase shift $\theta$ between the stimuli of the two oscillators $S_{1}$ and $S_{2}$ (Sec. II). For instance, in the phase-locked regime (Sec. V A) stimulation with a particular phase shift $\theta_{\max }$ leads to a nearly singular transient time (i.e., resynchronization time). Remarkably, this particular phase shift $\theta_{\max }$ is not equal to the value of the phase difference belonging to an unstable fixed point, as it is the case in phase oscillators coupled without delay [14-16]. Rather, slowest resynchronization is achieved for a $\theta_{\max }$, which resets the system of oscillators in a way that it gets trapped at an infinite-dimensional stable manifold $\mathcal{M}^{(s)}$, along which it creeps towards an (unstable) saddle point, from where it is eventually repelled towards the stable synchronized state. Put otherwise, the delay causes an infinite-dimensional dynamics, which leads to a completely different transient dynamics as compared to an oscillator model without delayed self-feedback. Slowest resynchronization, accompanied by maximal response clustering, can be elicited if the stimuli are tuned to the complex structure of the infinite-dimensional phase space.

From a medical standpoint this might be relevant. As shown above, an appropriate stimulus causes a particularly strong and long-lasting (but nevertheless transient) desynchronization due to a coupling with delayed self-feedback. Dynamical effects caused by delays involved in the interaction of oscillators may, hence, be exploited for control purposes. However, in an experimental or medical application it is typically difficult or impossible to tune a phase shift $\theta$ of the stimulation mechanism [see Eq. (2)]. Rather, for an experimental application the timing pattern of stimulus administration has to be chosen appropriately. More precisely, in order to induce a long-lasting transient desynchronization of oscillators, one has to administer identical stimuli $\left(S_{1}=S_{2}\right.$, i.e., $\theta=0$ ) at different times, with appropriately chosen delay in between (see also Refs. $[14,15,68]$ ). In phase oscillators without delay in the coupling, it has been shown that simultaneous stimulation of two oscillators with stimuli $S_{1}$ and $S_{2}$ with a phase shift $\theta_{\max }$ of $S_{2}$ can be approximated by delivering identical stimuli at different times, with a time shift $\tau_{\max }$ corresponding to $\theta_{\max }$, i.e., $\tau_{\max }=\theta_{\max } / T$, where $T$ is the (mean) period of the oscillators (see [15]). In this way, our stimulation approach can be applied to an experimental system, too. The overall goal of our study is to contribute not only to a better understanding of the mechanisms of transient and complex stimulus-locked responses, but also to the design of highly effective, but nevertheless mild control techniques for neural activity. Apart from medical applications one may envisage interesting applications to neuroscience, biology, and physics, where, e.g., the functional role of oscillations and synchronization processes [69-73] might be further clarified by perturbing these processes with suitable pulsatile stimuli.

\section{ACKNOWLEDGMENTS}

This study was supported by the Network of Excellence in Biosimulation (Grant no. 005137) and by IBM.

\section{APPENDIX: STIMULUS LOCKING INDICES}

To quantify the extend of stimulus locking of the variables $\psi_{1,2}$ and $\varphi_{1,2}$ of system (1), (2), following Refs. [14-18] we consider time-dependent stimulus locking indices

$$
\lambda_{\nu}[x(t)]=\left|\frac{1}{N} \sum_{k=1}^{N} e^{i \nu x^{(k)}(t)}\right| .
$$

The time $t$ here is the time relative to stimulus onset within each stimulation window around stimulus onset. So, each window has a time axis $t$, where the onset of the stimulus lies in $t=0$. The averaging is performed over $N$ stimulation trials, i.e., $k$ is the stimulus number. The variable $x(t)$ in Eq. (A1)) is one of $\psi_{1}, \psi_{2}, \varphi_{1}$, or $\varphi_{2}$ and $x^{(k)}(t)$ indicates the corresponding variable in the stimulation window around the $k$ th stimulus. Integer $\nu$ is the order of indices corresponding to the $\nu$ th Fourier mode of the phase distributions over trials, which reflects an emergence of a $\nu$-cluster state of the stimulus-induced system responses.

Further, we define resetting indices

$$
\begin{gathered}
\rho_{j}(t)=\lambda_{1}\left[\psi_{j}(t)\right], \\
\sigma_{j}(t)=\lambda_{1}\left[\varphi_{j}(t)\right], \quad j=1,2,
\end{gathered}
$$

and clustering indices

$$
\begin{gathered}
\alpha_{j}(t)=\lambda_{2}\left[\psi_{j}(t)\right]-\lambda_{1}\left[\psi_{j}(t)\right], \\
\delta_{j}(t)=\lambda_{2}\left[\varphi_{j}(t)\right]-\lambda_{1}\left[\varphi_{j}(t)\right], \quad j=1,2 .
\end{gathered}
$$

One can see that the resetting indices attain large values close to 1 if the distribution of the corresponding variable of system (1), (2) over stimulation trials exhibits a single sharp peak. On the other hand, for two peaks in the distribution with distance between them close to $\pi$, the resetting indices will be small, whereas the second order indices $\lambda_{2}$ will be large. The clustering indices will thus be also large indicating a two-cluster state of the distribution of the corresponding variable. For the higher-order clustering states up to a uniform distribution of the phases, both indices $\lambda_{1}$ and $\lambda_{2}$ are small and so are the resetting and clustering indices. 
[1] A. T. Winfree, The Geometry of Biological Time (SpringerVerlag, Berlin, 1980).

[2] L. Glass and M. C. MacKey, From Clocks to Chaos: The Rhythms of Life (Princeton University Press, Princeton, 1988).

[3] J. D. Murray, Mathematical Biology (Springer-Verlag, Berlin, 1990).

[4] P. A. Tass, Phase Resetting in Medicine and Biology: Stochastic Modelling and Data Analysis (Springer, Berlin, 1999).

[5] M. Hämäläinen, R. Hari, R. J. Ilmoniemi, J. Knuutila, and O. V. Lounasmaa, Rev. Mod. Phys. 65, 413 (1993).

[6] R. Hari and R. Salmelin, Trends Neurosci. 20, 44 (1997).

[7] K. H. Chiappa, Evoked Potentials in Clinical Medicine (Raven, New York, 1983).

[8] G. D. Dawson, Electroencephalogr. Clin. Neurophysiol. 44, 153 (1954).

[9] E. Basar, EEG-brain dynamics: Relation Between EEG and Brain Evoked Potentials (Elsevier, New York, 1980).

[10] B. M. Sayers, H. A. Beagley, and W. R. Henshall, Nature (London) 247, 481 (1974).

[11] S. Makeig, M. Westerfield, T. P. Jung, S. Enghoff, J. Townsend, E. Courchesne, and T. J. Sejnowski, Science 295, 690 (2002).

[12] B. W. Jervis, M. J. Nichols, T. Johnson, E. Allen, and N. R. Hudson, IEEE Trans. Biomed. Eng. 30, 43 (1983).

[13] C. Tallon-Baudry, O. Bertrand, C. Delpuech, and J. Pernier, J. Neurosci. 16, 4240 (1996).

[14] P. A. Tass, Europhys. Lett. 59, 199 (2002).

[15] P. A. Tass, Phys. Rev. E 67, 051902 (2003).

[16] P. A. Tass, Chaos 13, 364 (2003).

[17] P. A. Tass, Phys. Rev. E 69, 051909 (2004).

[18] P. A. Tass, Biol. Cybern. 91, 203 (2004).

[19] C. M. Marcus and R. M. Westervelt, Phys. Rev. A 39, 347 (1989).

[20] A. Beuter, J. Bélair, and C. Labrie, Bull. Math. Biol. 55, 525 (1993).

[21] J. Faro and S. Velasco, Physica D 110, 313 (1997).

[22] M. Yoshioka and M. Shiino, Phys. Rev. E 58, 3628 (1998).

[23] U. Ernst, K. Pawelzik, and T. Geisel, Phys. Rev. E 57, 2150 (1998).

[24] S. M. Crook, G. B. Ermentrout, M. C. Vanier, and J. M. Bower, J. Comput. Neurosci. 4, 161 (1997).

[25] S. Kunec and A. Bose, Phys. Rev. E 63, 021908 (2001).

[26] N. Burić and D. Todorović, Chaos, Solitons Fractals 13, 645 (2002).

[27] J. Fort and V. Méndez, Phys. Rev. Lett. 89, 178101 (2002).

[28] H. G. Schuster and P. Wagner, Prog. Theor. Phys. 81, 939 (1989).

[29] U. Ernst, K. Pawelzik, and T. Geisel, Phys. Rev. Lett. 74, 1570 (1995).

[30] S. Kim, S. H. Park, and C. S. Ryu, Phys. Rev. Lett. 79, 2911 (1997).

[31] M. K. Stephen Yeung and S. H. Strogatz, Phys. Rev. Lett. 82, 648 (1999).

[32] D. V. R. Reddy, A. Sen, and G. L. Johnston, Physica D 144, 335 (2000).

[33] D. V. Ramana Reddy, A. Sen, and G. L. Johnston, Phys. Rev. Lett. 85, 3381 (2000).

[34] M. G. Rosenblum and A. S. Pikovsky, Phys. Rev. Lett. 92, 114102 (2004)

[35] M. Rosenblum and A. Pikovsky, Phys. Rev. E 70, 041904
(2004).

[36] O. Popovych, V. Krachkovskyi, and P. A. Tass, in Proceedings of the 11th Workshop on Nonlinear Dynamics of Electronic Systems (NDES'2003), edited by R. Stoop (University of Zürich, Zürich, 2003), pp. 197-200.

[37] F. M. Atay, Phys. Rev. Lett. 91, 094101 (2003).

[38] A. Blanchard, Phase-locked Loops (John Wiley \& Sons, New York, 1976).

[39] W. Wischert, A. Wunderlin, A. Pelster, M. Olivier, and J. Groslambert, Phys. Rev. E 49, 203 (1994).

[40] P. Tass, A. Wunderlin, and M. Schanz, J. Biol. Phys. 21, 83 (1995).

[41] F. M. Gardner, Phaselock Techniques (Wiley, New York, 1979).

[42] F. C. Hoppensteadt, An Introduction to the Mathematics of Neurons (Cambridge University Press, Cambridge, 1986).

[43] E. Ahissar and E. Vaadia, Proc. Natl. Acad. Sci. U.S.A. 87, 8935 (1990).

[44] E. Ahissar, Neural Comput. 10, 597 (1998).

[45] J. Gordon, in Principles of Neural Science, 3rd ed., edited by E. R. Kandel, J. H. Schwartz, and T. M. Jessel (Elsevier, New York, 1991), pp. 580-595.

[46] E. Ahissar, S. Haidarliu, and M. Zacksenhouse, Proc. Natl. Acad. Sci. U.S.A. 94, 11633 (1997).

[47] E. Ahissar and A. Arieli, Neuron 32, 185 (2001).

[48] E. Ahissar and M. Zacksenhouse, Prog. Brain Res. 130, 75 (2001).

[49] O. V. Popovych, V. Krachkovskyi, and P. A. Tass (unpublished).

[50] M. G. Rosenblum, A. S. Pikovsky, and J. Kurths, Phys. Rev. Lett. 76, 1804 (1996).

[51] Y. Kuramoto, Chemical Oscillations, Waves, and Turbulence (Springer, Berlin, 1984).

[52] S. H. Strogatz, Physica D 143, 1 (2000).

[53] Y. Maistrenko, O. Popovych, O. Burylko, and P. A. Tass, Phys. Rev. Lett. 93, 084102 (2004).

[54] C. Ghez, in Principles of Neural Science, 3rd ed., edited by E. R. Kandel, J. H. Schwartz, and T. M. Jessell (Appleton and Lange, Norwalk, 1991), pp. 626-646.

[55] P. Tass, J. Kurths, M. G. Rosenblum, G. Guasti, and H. Hefter, Phys. Rev. E 54, R2224 (1996).

[56] G. D. VanWiggeren and R. Roy, Science 279, 1198 (1998).

[57] G. Kozyreff, A. G. Vladimirov, and P. Mandel, Phys. Rev. Lett. 85, 3809 (2000).

[58] K. Pyragas, Phys. Lett. A 170, 421 (1992).

[59] D. V. Ramana Reddy, A. Sen, and G. L. Johnston, Phys. Rev. Lett. 80, 5109 (1998).

[60] W. Just, S. Popovich, A. Amann, N. Baba, and E. Schöll, Phys. Rev. E 67, 026222 (2003).

[61] N. B. Janson, A. G. Balanov, and E. Schöll, Phys. Rev. Lett. 93, 010601 (1994).

[62] T. Probst, H. Plendl, W. Paulus, E. R. Wist, and M. Scherg, Exp. Brain Res. 93, 345 (1993).

[63] K. Hatanaka, N. Nakasato, K. Seki, A. Kanno, K. Mizoi, and T. Yoshimoto, Tokushima J. Exp. Med. 182, 9 (1997).

[64] A. Nakamura, R. Kakigi, M. Hoshiyama, S. Koyama, Y. Kitamura, and M. Shimojo, Brain Res. Cognit. Brain Res. 6, 9 (1997).

[65] U. B. Barnikol, K. Amunts, J. Dammers, H. Mohlberg, T. Fieseler, A. Malikovic, K. Zilles, M. Niedeggen, and P. A. Tass, Neuroimage (to be published). 
[66] P. A. Tass and M. Majtanik, Biol. Cybern. 94, 58 (2006).

[67] P. A. Tass, C. Hauptmann, and O. V. Popovych, Int. J. Bif. Chaos (to be published).

[68] P. A. Tass, Biol. Cybern. 89, 81 (2003).

[69] W. Singer, Annu. Rev. Physiol. 55, 349 (1993).

[70] A. B. Neiman and D. F. Russell, Phys. Rev. Lett. 88, 138103 (2002).
[71] P. Ashwin and M. Timme, Nonlinearity 18, 2035 (2005).

[72] P. Ashwin and M. Timme, Nature (London) 436, 36 (2005).

[73] Y. Zhai, I. Z. Kiss, P. A. Tass, and J. L. Hudson, Phys. Rev. E 71, 065202(R) (2005).

[74] The fixed points of system (4) always have a zero eigenvalue corresponding to the invariance of the trajectories with respect to a constant shift of the variable $\varphi_{2}$, see Eq. (5). 